TRANSACTIONS OF THE

AMERICAN MATHEMATICAL SOCIETY

Volume 365, Number 12, December 2013, Pages 6309-6341

S 0002-9947(2013)05834-5

Article electronically published on June 4, 2013

\title{
DELOCALIZED CHERN CHARACTER FOR STRINGY ORBIFOLD K-THEORY
}

\author{
JIANXUN HU AND BAI-LING WANG
}

\begin{abstract}
In this paper, we define a stringy product on $K_{\text {orb }}^{*}(\mathfrak{X}) \otimes \mathbb{C}$, the orbifold K-theory of any almost complex presentable orbifold $\mathfrak{X}$. We establish that under this stringy product, the delocalized Chern character

$$
\text { ch } h_{\text {deloc }}: K_{\text {orb }}^{*}(\mathfrak{X}) \otimes \mathbb{C} \longrightarrow H_{C R}^{*}(\mathfrak{X}),
$$

after a canonical modification, is a ring isomorphism. Here $H_{C R}^{*}(\mathfrak{X})$ is the Chen-Ruan cohomology of $\mathfrak{X}$. The proof relies on an intrinsic description of the obstruction bundles in the construction of the Chen-Ruan product. As an application, we investigate this stringy product on the equivariant K-theory $K_{G}^{*}(G)$ of a finite group $G$ with the conjugation action. It turns out that the stringy product is different from the Pontryagin product (the latter is also called the fusion product in string theory).
\end{abstract}

\section{INTRODUCTION}

The notion of an orbifold was first introduced by Satake under the name Vmanifold, and there have been many very interesting developments since its inception. For example, Kawasaki's orbifold index theory has been applied extensively in the study of geometric quantizations and in the development of orbifold string theory in quantum physics.

Recall that an orbifold $\mathfrak{X}$ is a paracompact Hausdorff space $X$ equipped with a compatible system of orbifold atlases locally modeled on quotient spaces of Euclidean spaces by finite group actions. For each $x \in X$, there is a neighbourhood $U_{x}$ and a homeomorphism $U_{x} \cong \tilde{U}_{x} / G_{x}$. $\mathfrak{X}$ is called effective if each local group $G_{x}$ acts on $\tilde{U}_{x}$ effectively. For any orbifold $\mathfrak{X}$, the de Rham cohomology $H_{d R}^{*}(\mathfrak{X})$ is well defined and, by a theorem of Satake [41, is isomorphic to the singular cohomology of the underlying space $X=|\mathfrak{X}|$.

Associated to an effective orbifold $\mathfrak{X}$ we have a canonical non-effective orbifold, called the inertia orbifold $\tilde{\mathfrak{X}}$ of $\mathfrak{X}$. The inertia orbifold $\tilde{\mathfrak{X}}$ consists of connected components of different dimensions (see page 7 in [14]),

$$
\tilde{\mathfrak{X}}=\bigsqcup_{(g)} \mathfrak{X}_{(g)},
$$

where $(g) \in \mathcal{T}_{1}$, the set of equivalence classes of conjugacy classes in local groups. Each $\mathfrak{X}_{(g)}$ is called a twisted sector of $\mathfrak{X}$ and is a sub-orbifold of $\mathfrak{X}$. The underlying

Received by the editors October 5, 2011 and, in revised form, March 19, 2012.

2010 Mathematics Subject Classification. Primary 57R19, 19L10, 22A22; Secondary 55N15, $53 \mathrm{D} 45$.

Key words and phrases. Orbifold K-theory, delocalized Chern character, Chen-Ruan cohomology. 
topological space of $\tilde{\mathfrak{X}}$, denoted by $|\tilde{\mathfrak{X}}|$, is the disjoint union of $X$ and the singularity set

$$
\Sigma X=\left\{(x,(g)) \mid x \in X, G_{x} \neq\{1\},(g) \in \operatorname{Conj}\left(G_{x}\right)\right\},
$$

where $\operatorname{Conj}\left(G_{x}\right)$ denotes the set of conjugacy classes in $G_{x}$.

In the development of Gromov-Witten theory for symplectic orbifolds, Chen and Ruan in [14] discovered a remarkable new cohomology theory on any almost complex orbifold $\mathfrak{X}$, called the Chen-Ruan cohomology $H_{C R}^{*}(\mathfrak{X})$. Almost complex orbifolds are those orbifolds with local models given by a finite group acting unitarily on complex spaces. The Chen-Ruan cohomology $H_{C R}^{*}(\mathfrak{X})$, as a classical limit of an orbifold quantum cohomology, is a cohomology of the inertia orbifold $\tilde{\mathfrak{X}}$

$$
H_{C R}^{*}(\mathfrak{X})=\left(H_{d R}^{*}(\tilde{\mathfrak{X}}, \mathbb{C}), \circ_{C R}\right)
$$

with a new product ${ }^{\circ} C R$, utilising the obstruction bundles over the moduli spaces of stable pseudo-holomorphic orbifold curves in $\mathfrak{X}$. The obstruction bundle $E^{[2]}$ in the construction of the Chen-Ruan product is a complex orbifold vector bundle

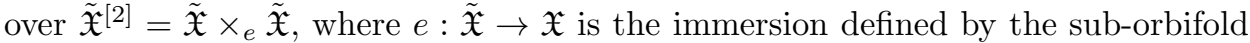
structure on each connected component of $\tilde{\mathfrak{X}}$. The associativity of the Chen-Ruan product follows from a property of the obstruction bundles discovered by ChenRuan in 14] using the gluing construction in Gromov-Witten theory.

For a compact almost complex orbifold $\mathfrak{X}$, Adem, Ruan and Zhang in 4 ] defined a stringy product on $K_{\text {orb }}^{*}(\tilde{\mathfrak{X}}, \tau)$, the twisted K-theory of the inertia orbifold $\tilde{\mathfrak{X}}$ with a transgressive twisting $\tau$. This product will be called the Adem-Ruan-Zhang product, denoted by $\bullet_{A R Z}$. For an orbifold $\mathfrak{X}$ arising from a smooth, projective variety $M$ with an action of a finite group $G$ or a Deligne-Mumford stack, an analogous product $\bullet_{J K K}$ was defined by Jarvis, Kaufmann and Kimura in 24 . on the untwisted orbifold K-theory of $\tilde{\mathfrak{X}}$. The $\operatorname{ring}\left(K_{\text {orb }}^{*}(\tilde{\mathfrak{X}}), \bullet_{J K K}\right)$ is called the full orbifold K-theory in 24]. This full orbifold K-theory was generalized to any compact abelian quotient orbifold $\mathfrak{X}$ by Becerra and Uribe in [10. The associated stringy product will be called the Becerra-Uribe product, denoted by $\bullet B U$. They also established an isomorphism

$$
\left(K_{\text {orb }}^{*}(\tilde{\mathfrak{X}}), \bullet_{B U}\right) \cong\left(K_{\text {orb }}^{*}(\tilde{\mathfrak{X}}), \bullet_{A R Z}\right) .
$$

In 10, Becerra and Uribe also established a ring homomorphism between the orbifold K-theory of $\tilde{\mathfrak{X}}$ and the Chen-Ruan cohomology under a modified Chern character map

$$
\widetilde{c h}:\left(K_{\text {orb }}^{*}(\tilde{\mathfrak{X}}), \bullet_{B U}\right) \longrightarrow\left(H_{C R}^{*}(\mathfrak{X}),{ }_{C R}\right) .
$$

We remark that this Chern character map is not an isomorphism over the complex coefficients. The full orbifold K-theory $\left(K_{\text {orb }}^{*}(\tilde{\mathfrak{X}}), \bullet_{J K K}\right)$ was further studied by Goldin, Harada, Holm and Kimura for abelian symplectic quotients in [20]. They gave a complete description of the ring structure of the full orbifold K-theory of weighted projective spaces obtained as symplectic quotients of $\mathbb{C}^{n}$ by weighted $S^{1}$ actions.

It is known that there is a delocalized Chern character (cf. 8 for proper actions of discrete groups and [12] for étale groupoids)

$$
\text { ch deloc }: K_{\text {orb }}^{*}(\mathfrak{X}) \longrightarrow H^{*}(\tilde{\mathfrak{X}}, \mathbb{C})=\bigoplus_{(g)} H^{*}\left(\mathfrak{X}_{(g)}, \mathbb{C}\right)
$$


relating the orbifold K-theory $K_{\text {orb }}^{*}(\mathfrak{X})$ to the 2-periodic de Rham cohomology of the inertia orbifold $\tilde{\mathfrak{X}}$. In this paper, we define a stringy product on the orbifold K-theory $K_{\text {orb }}^{*}(\mathfrak{X})$ of an almost complex compact orbifold $\mathfrak{X}$. The main result of this paper is that after a canonical modification, $c h_{\text {deloc }}$ is an isomorphism over the complex coefficients and sends the stringy product on $K_{\text {orb }}^{*}(\mathfrak{X})$ to the Chen-Ruan cup product on $H_{d R}^{*}(\tilde{\mathfrak{X}}, \mathbb{C})$.

The construction of this modified $c h_{\text {deloc }}$ relies on an intrinsic description of the obstruction bundle for the Chen-Ruan product and Adem-Ruan-Zhang's stringy product. Associated to the orbifold immersion

$$
e=\bigsqcup_{(g)} e_{(g)}: \tilde{\mathfrak{X}}=\bigsqcup_{(g)} \mathfrak{X}_{(g)} \rightarrow \mathfrak{X}
$$

there is a 2-sector orbifold $\tilde{\mathfrak{X}}^{[2]}=\tilde{\mathfrak{X}} \times_{e} \tilde{\mathfrak{X}}$, which consists of a disjoint union of sub-orbifolds of $\mathfrak{X}$,

$$
\bigsqcup_{\left(g_{1}, g_{2}\right)} \mathfrak{X}_{\left(g_{1}, g_{2}\right)},
$$

each of which is labelled by an equivalence class of conjugacy pairs in local groups. Consider the following commutative diagram:

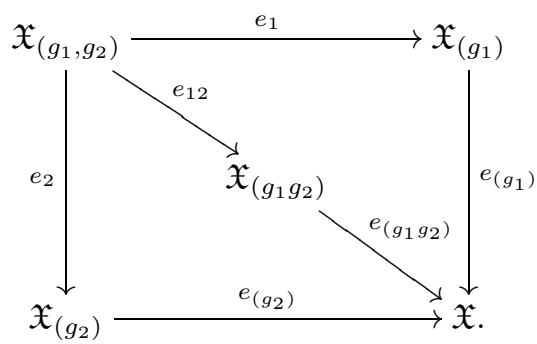

Then the obstruction bundle $E^{[2]}$ is a complex orbifold vector bundle over $\tilde{\mathfrak{X}}^{[2]}$ whose component over each $\mathfrak{X}_{\left(g_{1}, g_{2}\right)}$ is denoted by $E_{\left(g_{1}, g_{2}\right)}^{[2]}$ (see [14] for its definition).

Over the inertia orbifold $\tilde{\mathfrak{X}}$ there is an orbifold complex vector bundle

$$
\mathcal{N}_{e}=\bigsqcup_{(g)} \mathcal{N}_{(g)} \longrightarrow \bigsqcup_{(g)} \mathfrak{X}_{(g)}
$$

given by the orbifold normal bundle of each $\mathfrak{X}_{(g)}$ in $\mathfrak{X}$, each of which admits an automorphism of finite order. We can choose a Hermitian metric on the tangent bundle of $\mathfrak{X}$ such that the automorphism $\Phi$ acts unitarily on $\mathcal{N}_{e}$. Then each $\mathcal{N}_{(g)}$ has an eigen-bundle decomposition

$$
\mathcal{N}_{(g)}=\bigoplus_{\theta_{(g)} \in \mathbb{Q} \cap(0,1)} \mathcal{N}\left(\theta_{(g)}\right),
$$

where $\Phi$ on $\mathcal{N}\left(\theta_{(g)}\right)$ is multiplication by $e^{2 \pi \sqrt{-1} \theta_{(g)}}$ with $\theta_{(g)} \in \mathbb{Q} \cap(0,1)$. Define

$$
\mathcal{N}_{e, \Phi}=\bigoplus_{(g)} \sum_{\theta_{(g)}} \theta_{(g)} \mathcal{N}\left(\theta_{(g)}\right), \quad \mathcal{N}_{e, \Phi^{-1}}=\bigoplus_{(g)} \sum_{\theta_{(g)}}\left(1-\theta_{(g)}\right) \mathcal{N}\left(\theta_{(g)}\right)
$$

as linear combinations of vector bundles with rational coefficients or as elements in

$$
K_{\text {orb }}^{0}(\tilde{\mathfrak{X}}) \otimes \mathbb{Q}=\bigoplus_{(g)} K_{\text {orb }}^{0}\left(\mathfrak{X}_{(g)}\right) \otimes \mathbb{Q} .
$$


Theorem 1.1. Given an almost complex orbifold $\mathfrak{X}$, let $\mathcal{N}$ be the normal bundle of $\tilde{\mathfrak{X}}^{[2]}$ in $\mathfrak{X}$. Then the obstruction bundle $E^{[2]}$ in [14 satisfies the following identity:

$$
E^{[2]} \oplus \mathcal{N}=e_{1}^{*} \mathcal{N}_{e, \Phi}+e_{2}^{*} \mathcal{N}_{e, \Phi}+e_{12}^{*} \mathcal{N}_{e, \Phi^{-1}}
$$

in $K^{0}\left(\tilde{\mathfrak{X}}^{[2]}\right) \otimes \mathbb{Q}=\bigoplus_{\left(g_{1}, g_{2}\right)} K_{\text {orb }}^{0}\left(\mathfrak{X}_{\left(g_{1}, g_{2}\right)}\right) \otimes \mathbb{Q}$.

Theorem 1.1 implies that the linear combination of a vector bundle with rational coefficients

$$
e_{1}^{*} \mathcal{N}_{e, \Phi}+e_{2}^{*} \mathcal{N}_{e, \Phi}+e_{12}^{*} \mathcal{N}_{e, \Phi^{-1}}-\mathcal{N}
$$

after combining like terms, is a genuine vector bundle, which can be identified with the obstruction bundle $E^{[2]}$. This theorem was obtained in [13 for abelian orbifolds and in 24] for smooth Deligne-Mumford stacks. In this paper, we give a direct proof of this theorem using an equivariant version of Kawasaki's orbifold index theorem. We also employ this theorem to give an intrinsic definition of ChenRuan cohomology as Chen and Hu did for abelian orbifolds 13 .

The modified delocalized Chern character is given by

$$
\widetilde{c h}_{\text {deloc }}=\mathfrak{T}\left(\mathcal{N}_{e}, \Phi\right) \wedge \text { ch deloc }: K_{\text {orb }}^{*}(\mathfrak{X}) \longrightarrow \bigoplus_{(g)} H^{*}\left(\mathfrak{X}_{(g)}, \mathbb{C}\right),
$$

where on each component $\mathfrak{X}_{(g)}, \mathfrak{T}\left(\mathcal{N}_{e}, \Phi\right)$ is defined by

$$
\prod_{\theta_{(g)}} \mathfrak{T}\left(\mathcal{N}\left(\theta_{(g)}\right)\right)^{\theta_{(g)}} \in H^{*}\left(\mathfrak{X}_{(g)}\right),
$$

associated to the eigen-bundle decomposition (1.1). Here $\mathfrak{T}(V)^{m}$ is the multiplicative characteristic class of an orbifold complex vector bundle $V$ corresponding to the formal power series $\left(\frac{1-e^{x}}{x}\right)^{m}$ for $m \in \mathbb{Q} \cap(0,1)$.

Theorem 1.2. Let $\mathfrak{X}$ be a compact, almost complex, effective orbifold. Then there is an associative product $\circ$ on the orbifold $K$-theory $K_{\text {orb }}^{*}(\mathfrak{X}) \otimes \mathbb{C}$ such that the modified delocalized Chern character

$$
\widetilde{c h}_{\text {deloc }}:\left(K_{\text {orb }}^{*}(\mathfrak{X}) \otimes \mathbb{C}, \circ\right) \longrightarrow\left(H_{C R}^{*}(\mathfrak{X}, \mathbb{C}), *_{C R}\right)
$$

is a ring isomorphism.

In Section 2, we review some of the basics of orbifold, orbifold K-theory and orbifold index theorem used in this paper. In Section 3, we give an intrinsic definition of the Chen-Ruan cohomology after we establish Theorem 1.1. In Section 4, we define the stringy product on orbifold K-theory of an almost complex compact orbifold and prove Theorem 1.2. We also compute a few examples in Section 4 and discover that the stringy product on the orbifold K-theory of the orbifold $[G / G]$ (obtained from the conjugation of a finite group on itself) is different from the Pontrjagin product on $K_{G}(G)$. In Section 5 , we briefly discuss the stringy product on twisted orbifold K-theory for orbifolds with torsion twistings.

\section{REVIEW OF ORBIFOLDS AND ORBIFOLD INDEX THEORY}

In this section, we will give a brief review of the notion of orbifolds in terms of an orbifold atlas and proper étale groupoids. Then we review the orbifold index theory using delocalized Chern character. The main references for this section are [2, 25], 28, 30] and 42. 
2.1. Orbifolds and orbifold groupoids. Let $X$ be a paracompact Hausdorff space. An $n$-dimensional orbifold chart for an open subset $U$ of $X$ is a triple $(\tilde{U}, G, \pi)$ given by a connected open subset $\tilde{U} \subset \mathbb{R}^{n}$, together with a smooth action of a finite group $G$ such that $\pi: \tilde{U} \rightarrow U$ is the induced quotient map. An orbifold chart $(\tilde{U}, G, \pi)$ is called effective if the action of $G$ on $\tilde{U}$ is effective. Given an inclusion $\iota_{i j}: U_{i} \hookrightarrow U_{j}$, an embedding of orbifold charts

$$
\left(\phi_{i j}, \lambda_{i j}\right):\left(\tilde{U}_{i}, G_{i}, \pi_{i}\right) \hookrightarrow\left(\tilde{U}_{j}, G_{j}, \pi_{j}\right)
$$

consists of an injective group homomorphism $\lambda_{i j}: G_{i} \rightarrow G_{j}$ and an embedding $\phi_{i j}: \tilde{U}_{i} \hookrightarrow \tilde{U}_{j}$ covering the inclusion $\iota_{i j}$ such that $\phi_{i j}$ is $G_{i}$-equivariant with respect to $\phi_{i j}$, that is,

$$
\phi_{i j}(g x)=\lambda_{i j}(g) \phi_{i j}(x),
$$

for $x \in \tilde{U}_{i}$ and $g \in G_{i}$. In the non-effective case, we further require that the subgroup of $G_{i}$ acting trivially on $U_{i}$ is isomomorphically mapped to the subgroup of $G_{j}$ acting trivially on $U_{j}$. Whenever $U_{i} \subset U_{j} \subset U_{k}$, there exists an element $g \in G_{k}$ such that

$$
g \circ \phi_{i k}=\phi_{j k} \circ \phi_{i j}, \quad g \lambda_{i k} g^{-1}=\lambda_{j k} \circ \lambda_{i j}
$$

Definition 2.1. An orbifold atlas on $X$ is a collection of orbifold charts $\mathcal{U}=$ $\left\{\left(\tilde{U}_{i}, G_{i}, \pi_{i}\right)\right\}$ for an open covering $\left\{U_{i}\right\}$ of $X$ such that

(1) $\left\{U_{i}\right\}$ is closed under finite intersection.

(2) Given any inclusion $U_{i} \subset U_{j}$, there is an embedding of orbifold charts $\left(\phi_{i j}, \lambda_{i j}\right):\left(\tilde{U}_{i}, G_{i}, \pi_{i}\right) \hookrightarrow\left(\tilde{U}_{j}, G_{j}, \pi_{j}\right)$.

Two orbifold atlases $\mathcal{U}$ and $\mathcal{V}$ are equivalent if there is a common orbifold atlas $\mathcal{W}$ refining $\mathcal{U}$ and $\mathcal{V}$. An orbifold $\mathfrak{X}=(X, \mathcal{U})$ is a paracompact Hausdorff space $X$ with an equivalence class of orbifold atlases. Given an orbifold $\mathfrak{X}=(X, \mathcal{U})$ and a point $x \in X$, let $(\tilde{U}, G, \pi)$ be an orbifold chart around $x$. Then the local group at $x$ is defined to be the stabilizer of $\tilde{x} \in \pi^{-1}(x)$, which is uniquely defined up to conjugation.

The notion of an orbifold and many of its invariants can be reformulated using the language of groupoids. For general orbifolds, the groupoid viewpoint is also essential for the $C^{*}$-algebraic definition of K-theory and its twisted version in order to get a cohomology theory satisfying the Mayer-Vietoris axiom. We briefly recall the definition of groupoids and their roles in orbifold theory.

A Lie groupoid $\mathcal{G}=\left(\mathcal{G}_{1} \rightrightarrows \mathcal{G}_{0}\right)$ consists of two smooth manifolds $\mathcal{G}_{1}$ and $\mathcal{G}_{0}$, together with five smooth maps $(s, t, m, u, i)$ satisfying the following properties:

(1) The source map and the target map $s, t: \mathcal{G}_{1} \rightarrow \mathcal{G}_{0}$ are submersions.

(2) The composition map

$$
m: \mathcal{G}_{1}{ }_{t} \times_{s} \mathcal{G}_{1}=\left\{\left(g_{1}, g_{2}\right) \in \mathcal{G}_{1} \times \mathcal{G}_{1}: t\left(g_{1}\right)=s\left(g_{2}\right)\right\} \rightarrow \mathcal{G}_{1},
$$

written as $m\left(g_{1}, g_{2}\right)=g_{1} \cdot g_{2}$, satisfies the obvious associative property.

(3) The unit map $u: \mathcal{G}_{0} \rightarrow \mathcal{G}_{1}$ is a two-sided unit for the composition.

(4) The inverse map $i: \mathcal{G}_{1} \rightarrow \mathcal{G}_{1}, i(g)=g^{-1}$, is a two-sided inverse for the composition.

A Lie groupoid $\mathcal{G}=\left(\mathcal{G}_{1} \rightrightarrows \mathcal{G}_{0}\right)$ is proper if $(s, t): \mathcal{G}_{1} \rightarrow \mathcal{G}_{0} \times \mathcal{G}_{0}$ is proper and is called étale if $s$ and $t$ are local diffeomorphisms. 
Let $\mathcal{G}_{1} \rightrightarrows \mathcal{G}_{0}$ and $\mathcal{H}_{1} \rightrightarrows \mathcal{H}_{0}$ be two Lie groupoids. A generalized morphism between $\mathcal{G}$ and $\mathcal{H}$ is a right principal $\mathcal{H}$-bundle $P_{f}$ over $\mathcal{G}_{0}$ which is also a left $\mathcal{G}$ bundle over $\mathcal{H}_{0}$ such that the left $\mathcal{G}$-action and the right $\mathcal{H}$-action commute. This is formally denoted by

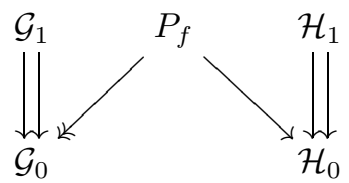

For example, a rank $k$ Hermitian vector bundle over a Lie groupoid $\mathcal{G}$ is defined by a $U(k)$-valued cocycle over $\mathcal{G}$, that is, a generalized morphism $\mathcal{G} \rightarrow U(k)$ where $U(k)$ is viewed as a Lie groupoid with one object. Note that generalized morphisms can be composed. This implies that the pull-back of a vector bundle over a groupoid by any generalized morphism is well defined. Note that a generalized morphism $f$ between $\mathcal{G}$ and $\mathcal{H}$ is invertible if $P_{f}$ in (2.1) is also a principal $\mathcal{G}$-bundle over $\mathcal{H}_{0}$. Then $\mathcal{G}$ and $\mathcal{H}$ are called Morita equivalent.

Remark 2.2. (1) As observed in 31] and [27], given an orbifold $\mathfrak{X}=(X, \mathcal{U})$, there is a canonical proper étale Lie groupoid $\mathcal{G}[\mathcal{U}]$, locally given by the action groupoid $\tilde{U}_{i} \rtimes G_{i} \rightrightarrows \tilde{U}_{i}$. For two equivalent orbifold atlases $\mathcal{U}$ and $\mathcal{V}, \mathcal{G}[\mathcal{U}]$ and $\mathcal{G}[\mathcal{V}]$ are Morita equivalent.

(2) Given a proper étale Lie groupoid $\mathcal{G}$, there is a canonical orbifold structure on its orbit space $|\mathcal{G}|$; see [30] and Proposition 1.44 in [2]. Two Morita equivalent proper étale Lie groupoids define the same orbifold up to isomorphism (Theorem 1.45 in [2]).

(3) Given an orbifold $\mathfrak{X}=(X, \mathcal{U})$, a proper étale Lie groupoid $\mathcal{G}$ is called a presentation of $\mathfrak{X}$ if there is a homeomorphism $f:|\mathcal{G}| \rightarrow X$ such that $f^{*} \mathcal{U}$ agrees with the canonical orbifold structure on $|\mathcal{G}|$. A proper étale Lie groupoid is also called an orbifold groupoid for simplicity.

(4) An orbifold is called effective if any local group acts effectively on its orbifold chart. For an $n$-dimensional effective orbifold $\mathfrak{X}=(X, \mathcal{U})$, the corresponding proper étale Lie groupoid is Morita equivalent to the action groupoid associated to the $O(n)$-action on the orthonormal frame bundle for a Riemannian metric on $\mathfrak{X}$.

Definition 2.3. Let $\mathfrak{X}$ be an orbifold with a presenting groupoid $\mathcal{G}$. If $|\mathcal{G}|$ is compact, the de Rham cohomology of an orbifold $\mathfrak{X}=(X, \mathcal{U})$, denoted by $H_{\text {orb }}^{*}(\mathfrak{X}, \mathbb{R})$, is defined to be the de Rham cohomology of $\mathcal{G}$ which is the cohomology of the $\mathcal{G}$-invariant de Rham complex $\left(\Omega^{p}(\mathcal{G}), d\right)$, where

$$
\Omega^{p}(\mathcal{G})=\left\{\omega \in \Omega^{p}\left(\mathcal{G}_{0}\right) \mid s^{*} \omega=t^{*} \omega\right\} .
$$

If $|\mathcal{G}|$ is not compact, the de Rham cohomology of $\mathfrak{X}$ is defined to be the de Rham cohomology with compact supports of $\mathcal{G}$, where a differential form $\omega \in \Omega^{p}(\mathcal{G})$ has compact support in $|\mathcal{G}|$.

An orbifold vector bundle $\mathcal{E}$ over an orbifold $\mathfrak{X}=(X, \mathcal{U})$ is a family of equivariant vector bundles

$$
\left\{\left(\tilde{E}_{i} \rightarrow \tilde{U}_{i}, G_{i}\right)\right\}
$$

such that for any embedding of orbifold charts $\phi_{i j}:\left(\tilde{U}_{i}, G_{i}\right) \hookrightarrow\left(\tilde{U}_{j}, G_{j}\right)$, there is a $G_{i}$-equivariant bundle map $\tilde{\phi}_{i j}: \tilde{E}_{i} \rightarrow \tilde{E}_{j}$ covering $\phi_{i j}: \tilde{U}_{i} \rightarrow \tilde{U}_{j}$. The total space 
$E=\bigcup\left(\tilde{E}_{i} / G_{i}\right)$ of an orbibundle $\mathcal{E} \rightarrow \mathfrak{X}$ has a canonical orbifold structure given by $\left\{\left(\tilde{E}_{i}, G_{i}\right)\right\}$. A connection $\nabla$ on an orbibundle $\mathcal{E} \rightarrow \mathfrak{X}$ is a family of invariant connections $\left\{\nabla_{i}\right\}$ on $\left\{\left(\tilde{E}_{i} \rightarrow \tilde{U}_{i}, G_{i}\right)\right\}$ which are compatible with the bundle maps $\left\{\tilde{\phi}_{i j}\right\}$. Examples of orbibundles over $\mathfrak{X}$ include its tangent bundle $T \mathfrak{X}$ and its cotangent bundle $T^{*} \mathfrak{X}$. In general, orbifold vector bundles over $\mathfrak{X}$ do not define vector bundles over the underlying topological space $X$.

Recall that a vector bundle over a Lie groupoid $\mathcal{G}=\left(\mathcal{G}_{1} \rightrightarrows \mathcal{G}_{0}\right)$ is a $\mathcal{G}$-vector bundle $E$ over $\mathcal{G}_{0}$, that is, a vector bundle $E$ with a fiberwise linear action of $\mathcal{G}$ covering the canonical action of $\mathcal{G}$ on $\mathcal{G}_{0}$. One can check that an orbibundle $\mathcal{E}=\left(E, \mathcal{U}_{E}\right)$ defines a canonical vector bundle over the groupoid $\mathcal{G}[\mathcal{U}]$.

Definition 2.4. Let $\mathfrak{X}$ be an orbifold with a presenting groupoid $\mathcal{G}$. If $|\mathcal{G}|$ is compact, the orbifold K-theory of $\mathfrak{X}$, denoted by $K_{\text {orb }}^{0}(\mathfrak{X})$, is defined to be the Grothendieck ring of isomorphism classes of vector bundles over $\mathcal{G}$. If $|\mathcal{G}|$ is not compact, the orbifold K-theory of $\mathfrak{X}$ is defined to be the Grothendieck ring of isomorphism classes of complex vector bundles with compact supports over $\mathcal{G}$. Here a vector bundle with compact support over $\mathcal{G}$ is a $\mathbb{Z}_{2}$-graded $\mathcal{G}$-equivariant complex vector bundle $E=E^{0} \oplus E^{1}$ with a $\mathcal{G}$-equivariant bundle morphism $\sigma: E_{0} \rightarrow E_{1}$ such that the support of $\sigma$

$$
\left\{x \in \mathcal{G}_{0} \mid \sigma_{x}: E_{x}^{0} \rightarrow E_{x}^{1} \text { is not an isomorphism }\right\}
$$

defines a compact set in $|\mathcal{G}|$.

The de Rham cohomology and the orbifold K-theory are well defined, as a Morita equivalence between two groupoids induces an isomorphism on de Rham cohomology and orbifold K-theory respectively. The Satake-de Rham theorem for an orbifold $\mathfrak{X}=(X, \mathcal{U})$ leads to an isomorphism

$$
H_{\text {orb }}^{*}(\mathfrak{X}, \mathbb{R}) \cong H^{*}(X, \mathbb{R})
$$

between the de Rham cohomology and the singular cohomology of the underlying topological space. The standard Chern-Weil construction applied to a compact orbifold $\mathfrak{X}$ gives rise to the Chern character map

$$
\text { ch }: K_{\text {orb }}^{0}(\mathfrak{X}) \longrightarrow H_{\text {orb }}^{\text {ev }}(\mathfrak{X}, \mathbb{C}),
$$

which is a ring homomorphism. Here the ring structure on $K_{\text {orb }}^{0}(\mathfrak{X})$ is given by the tensor product of vector bundles and the ring structure on $H_{\text {orb }}^{e v}(\mathfrak{X}, \mathbb{C})$ is given by the wedge product of differential forms. This Chern character over the complex coefficients is not an isomorphism.

Remark 2.5. The definition of orbifold K-theory can be extended in the usual way to a $\mathbb{Z}_{2}$-graded cohomology theory (see [2]),

$$
K_{\text {orb }}^{*}(\mathfrak{X})=K_{\text {orb }}^{0}(\mathfrak{X}) \oplus K_{\text {orb }}^{1}(\mathfrak{X}) .
$$

For an orbifold as a quotient of a compact Lie group action on locally compact manifolds with finite stabilizers, the orbifold K-theory has the usual Bott periodicity, the Mayer-Vietoris exact sequence and the Thom isomorphism for orbifold Spin $^{c}$ vector bundles.

We can define the orbifold K-theory of an orbifold $\mathfrak{X}$ as the K-theory of the reduced $C^{*}$-algebra $C_{\text {red }}^{*}(\mathfrak{X})$ of the canonical proper étale groupoid (see Chapter 2 in [15]). The $C^{*}$-algebraic orbifold K-theory is a module over the orbifold K-theory using orbifold vector bundles. If $\mathfrak{X}$ is presentable, that is, $\mathfrak{X}$ is the orbifold obtained 
from a locally free action of a compact Lie group $G$ on a smooth manifold $M$, then $C^{*}(\mathfrak{X})$ is Morita equivalent to the cross-product $C^{*}$-algebra $C(M) \rtimes G$. This Morita equivalence can be used to define an isomorphism between the orbifold $\mathrm{K}$ theory $K_{\text {orb }}^{*}(\mathfrak{X})$ defined in Definition 2.3 and the K-theory of the reduced $C^{*}$-algebra $C_{\text {red }}^{*}(\mathfrak{X})$ :

$$
K_{\text {orb }}^{*}(\mathfrak{X}) \cong K_{G}^{*}(M) \cong K_{*}(C(M) \rtimes G) \cong K_{*}\left(C_{\text {red }}^{*}(\mathfrak{X})\right) .
$$

Conjecturally, the isomorphism

$$
K_{\text {orb }}^{*}(\mathfrak{X}) \cong K_{*}\left(C_{\text {red }}^{*}(\mathfrak{X})\right)
$$

holds for general orbifolds.

2.2. Delocalized Chern character and the orbifold index theory. For any compact orbifold $\mathfrak{X}$, there is a delocalized Chern character,

$$
\text { ch deloc }: K_{\text {orb }}^{*}(\mathfrak{X}) \longrightarrow H^{*}(\tilde{\mathfrak{X}}, \mathbb{C}),
$$

from the orbifold K-theory of $\mathfrak{X}$ to the 2-periodic de Rham cohomology of its inertia orbifold $\tilde{\mathfrak{X}}$. It was defined in [8] for proper actions of discrete groups and in 12 for étale groupoids. The delocalized Chern character made its first appearance in the Lefschetz formulas of Atiyah-Bott in [5] and the Kawasaki orbifold index theorem in 25 .

Let $\mathfrak{X}=(X, \mathcal{U})$ be an orbifold. Then the set of pairs

$$
\tilde{X}=\left\{\left(x,(g)_{G_{x}}\right) \mid x \in X, g \in G_{x}\right\},
$$

where $(g)_{G_{x}}$ is the conjugacy class of $g$ in the local group $G_{x}$, has a natural orbifold structure given by

$$
\left\{\left(\tilde{U}^{g}, Z_{G}(g), \tilde{\pi}, \tilde{U}^{g} / C(g)\right) \mid g \in G\right\} .
$$

Here for each orbifold chart $(\tilde{U}, G, \pi, U) \in \mathcal{U}, Z_{G}(g)$ is the centralizer of $g$ in $G$ and $\tilde{U}^{g}$ is the fixed-point set of $g$ in $\tilde{U}$. This orbifold, denoted by $\tilde{\mathfrak{X}}$, is called the inertia orbifold of $\mathfrak{X}$. The inertia orbifold $\tilde{\mathfrak{X}}$ consists of a disjoint union of sub-orbifolds of $\mathfrak{X}$.

To describe the connected components of $\tilde{\mathfrak{X}}$, we need to introduce an equivalence relation on the set of conjugacy classes in local groups as in 14. For each $x \in X$, let $\left(\tilde{U}_{x}, G_{x}, \pi_{x}, U_{x}\right)$ be a local orbifold chart at $x$. If $y \in U_{x}$, then up to conjugation, there is an injective homomorphism of local groups $G_{y} \rightarrow G_{x}$. Hence the conjugacy class $(g)_{G_{x}}$ is well defined for $g \in G_{y}$. We define the equivalence to be generated by the relation $(g)_{G_{y}} \sim(g)_{G_{x}}$. Let $\mathcal{T}_{1}$ be the set of equivalence classes. Then

$$
\tilde{\mathfrak{X}}=\bigsqcup_{(g) \in \mathcal{T}_{1}} \mathfrak{X}_{(g)},
$$

where $\mathfrak{X}_{(g)}=\left\{\left(x,\left(g^{\prime}\right)_{G_{x}} \mid g^{\prime} \in G_{x},\left(g^{\prime}\right)_{G_{x}} \sim(g)\right\}\right.$. Note that $\mathfrak{X}_{(1)}=\mathfrak{X}$ is called the non-twisted sector and $\mathfrak{X}_{(g)}$ for $g \neq 1$ is called a twisted sector of $\mathfrak{X}$.

Let $\mathcal{G}$ be a proper étale Lie groupoid representing a compact orbifold $\mathfrak{X}=(X, \mathcal{U})$. Then the groupoid associated to the inertia orbifold $\tilde{\mathfrak{X}}$ is given by $\tilde{\mathcal{G}}=\left((s, t): \tilde{\mathcal{G}}_{1} \rightrightarrows\right.$ $\left.\tilde{\mathcal{G}}_{0}\right)$, where

$$
\tilde{\mathcal{G}}_{0}=\left\{g \in \mathcal{G}_{1} \mid s(g)=t(g)\right\}, \quad \tilde{\mathcal{G}}_{1}=\left\{(g, h) \in \mathcal{G}_{1} \times \mathcal{G}_{1} \mid g \in \tilde{\mathcal{G}}_{0}, t(g)=s(h)\right\},
$$

with the source map $s(g, h)=g$ and the target map $t(g, h)=h^{-1} g h$. There is an obvious evaluation map $e: \tilde{\mathcal{G}} \rightarrow \mathcal{G}$ which corresponds to the obvious orbifold 
immersion

$$
e=\bigsqcup e_{(g)}: \tilde{\mathfrak{X}}=\bigsqcup_{(g) \in \mathcal{T}_{1}} \mathfrak{X}_{(g)} \rightarrow \mathfrak{X} .
$$

Given a complex orbifold bundle $E$ over $\mathfrak{X}$ or a complex vector bundle over its presenting groupoid $\mathcal{G}$, the pull-back bundle $e^{*} E$ over $\tilde{\mathfrak{X}}$ or $\tilde{\mathcal{G}}$ has a canonical automorphism $\Phi$. With respect to a $\mathcal{G}$-invariant Hermitian metric on $E$, there is an eigen-bundle decomposition of $e^{*} E$,

$$
e^{*} E=\bigoplus_{\theta \in \mathbb{Q} \cap[0,1)} E_{\theta},
$$

where $E_{\theta}$ is a complex vector bundle over $\tilde{\mathcal{G}}$, on which $\Phi$ acts by multiplying $e^{2 \pi \sqrt{-1} \theta}$. Define

$$
\operatorname{ch}_{\text {deloc }}(E)=\sum_{\theta} e^{2 \pi \sqrt{-1} \theta} \operatorname{ch}\left(E_{\theta}\right) \in H^{e v}(\tilde{\mathcal{G}}, \mathbb{C}) \cong H_{\text {orb }}^{e v}(\tilde{\mathfrak{X}}, \mathbb{C}),
$$

where $\operatorname{ch}\left(E_{\theta}\right) \in H^{e v}(\tilde{\mathcal{G}}, \mathbb{C})$ is the ordinary Chern character of $E_{\theta}$.

The odd delocalized Chern character

$$
c h_{\text {deloc }}: K_{\text {orb }}^{1}(\mathfrak{X}) \longrightarrow H^{\text {odd }}(\tilde{\mathfrak{X}}, \mathbb{C})
$$

can be defined in the usual way. Using the standard compactly supported condition, the delocalized Chern character can be defined for non-compact orbifolds.

Remark 2.6. For a compact quotient orbifold $\mathfrak{X}=[M / G]$, where $G$ is a compact Lie group acting on a compact manifold $M$ with finite stablizers, Adem and Ruan obtained a decomposition (cf. Theorem 5.1 and Corollary 5.2 in [3] )

$$
K_{\text {orb }}^{*}(\mathfrak{X}) \otimes \mathbb{C} \cong K_{G}^{*}(M) \otimes \mathbb{C} \cong \bigoplus_{\{\text {conjugacy class }\langle g\rangle: g \in G\}} K^{*}\left(M^{g} / Z_{G}(g)\right) \otimes \mathbb{C},
$$

where the closed submanifold $M^{g}$ is the fixed point set of the $g$-action and $Z_{G}(g)$ is the centralizer of $g$ in $G$. Applying the ordinary Chern character on each $K^{*}\left(M^{g} / Z_{G}(g)\right)$, we get an alternative definition of the delocalized Chern character over $\mathbb{C}$ :

$$
c h_{\text {deloc }}: K_{G}^{*}(M) \otimes \mathbb{C} \longrightarrow \bigoplus_{\{\langle g\rangle: g \in G\}} H^{*}\left(M^{g} / Z_{G}(g), \mathbb{C}\right) .
$$

Going through the proof of Theorem 5.1 in [3], particularly the ring map from the representation ring of the cyclic subgroup $\langle g\rangle$ generated by $g$ to the the cyclotomic field $\mathbb{Q}\left(e^{2 \pi \sqrt{-1} /|(g)|}\right)$, one can check that the delocalized Chern character (2.2) agrees with the delocalized Chern character defined by eigen-bundle decompositions. Here we identify the inertia orbifold $\tilde{\mathfrak{X}}$ with the orbifold $\bigsqcup_{\langle g\rangle}\left[M^{g} / Z_{G}(g)\right]$.

Proposition 2.7. For any compact presentable orbifold $\mathfrak{X}$, the delocalized Chern character gives a ring isomorphism

$$
\text { ch }_{\text {deloc }}: K_{\text {orb }}^{*}(\mathfrak{X}) \otimes_{\mathbb{Z}} \mathbb{C} \longrightarrow H^{*}(\tilde{\mathfrak{X}}, \mathbb{C})
$$

over $\mathbb{C}$.

Proof. The proof follows from the isomorphism for orbifolds obtained from a finite group action on a locally compact manifold and the Mayer-Vietoris sequence for open covers. Recall that the canonical groupoid $\mathcal{G}$ associated to an orbifold chart $\left\{\left(\tilde{U}_{i}, G_{i}, \pi_{i}\right)\right\}$, when restricted to each open set $\tilde{U}_{i}$, is an action groupoid $\tilde{U}_{i} \rtimes$ 
$G_{i}$. With compactly supported K-theory and de Rham cohomology, one has the following isomorphism (Theorem 1.19 in [8]) of vector spaces over $\mathbb{C}$ :

$$
\text { ch deloc }: K_{G_{i}}^{*}\left(\tilde{U}_{i}\right) \otimes_{\mathbb{Z}} \mathbb{C} \longrightarrow H_{c}^{*}\left(\tilde{U}_{i}, G_{i}\right),
$$

where $H_{c}^{*}\left(\tilde{U}_{i}, G_{i}\right)=\left[\bigoplus_{g \in G_{i}} H_{c}^{*}\left(\tilde{U}_{i}^{g}, \mathbb{C}\right)\right]^{G_{i}}$ with $\tilde{U}_{i}^{g}$ the fixed point submanifold of the $g$-action. From the definition, we see that $H_{c}^{*}\left(\tilde{U}_{i}, G_{i}\right)$ is the de Rham cohomology of the inertia groupoid of the action groupoid $\tilde{U}_{i} \rtimes G_{i}$. By an induction argument, we can apply the Mayer-Vietoris sequence for open covers and the five lemma to show that

$$
\operatorname{ch}_{\text {deloc }}: K_{\text {orb }}^{*}(\mathfrak{X}) \otimes_{\mathbb{Z}} \mathbb{C} \longrightarrow H^{*}(\tilde{\mathfrak{X}}, \mathbb{C})
$$

is an isomorphism of vector spaces. For two vector bundles $E$ and $F$ with eigenbundle decompositions

$$
e^{*} E=\bigoplus_{\theta} E_{\theta}, \quad e^{*} F=\bigoplus_{\zeta} F_{\zeta}
$$

we have

$$
e^{*}(E \otimes F)=\bigoplus_{\theta, \zeta} E_{\theta} \otimes F_{\zeta},
$$

from which one immediately gets

$$
\operatorname{ch}_{\text {deloc }}(E \otimes F)=\operatorname{ch}_{\text {deloc }}(E) \cup \operatorname{ch}_{\text {deloc }}(F) .
$$

Hence, $h_{\text {deloc }}: K_{\text {orb }}^{0}(\mathfrak{X}) \otimes_{\mathbb{Z}} \mathbb{C} \longrightarrow H_{\text {orb }}^{\text {ev }}(\tilde{\mathfrak{X}}, \mathbb{C})$ is a ring isomorphism. The ring isomorphism for the odd delocalized Chern character can be proved by the standard desuspension argument.

Remark 2.8. The delocalized Chern character can be applied to write the Kawasaki orbifold index [25] as follows. Let $\mathfrak{X}$ be a compact almost complex orbifold with a Hermitian connection on the tangent bundle $T \tilde{\mathfrak{X}}$ of the inertia orbifold $\tilde{\mathfrak{X}}$. Let $E$ be a complex orbifold Hermitian vector bundle with a Hermitian connection. Let $\not D_{E}^{ \pm}$be the corresponding $S_{p i n}^{c}$ Dirac operator. The orbifold index formula in 25 ] can be expressed as

$$
\operatorname{Index}\left(\not D_{E}^{+}\right)=\int_{\tilde{\mathfrak{X}}}^{\text {orb }} \operatorname{ch} h_{\text {deloc }}(E) T d_{\text {deloc }}(\mathfrak{X}),
$$

where $T d_{\text {deloc }}(\tilde{\mathfrak{X}}) \in H^{*}(\tilde{\mathfrak{X}})$ is the delocalized Todd class of $\mathfrak{X}$ whose $\mathfrak{X}_{(g)}$-component is given by

$$
\frac{\operatorname{Td}\left(\mathfrak{X}_{(g)}\right)}{\operatorname{det}\left(1-g^{-1} e^{F_{\mathcal{N}_{(g)}} / 2 \pi i}\right)} .
$$

Here $\operatorname{Td}\left(\mathfrak{X}_{(g)}\right)$ is the Todd form of $\mathfrak{X}_{(g)}$ and $F_{\mathcal{N}_{(g)}}$ is the curvature of the induced Hermitian connection on the normal bundle $\mathcal{N}_{(g)}$. In this paper, we will use the equivariant orbifold index formula for a finite group $H$ acting trivially on $\mathfrak{X}$. Here we briefly discuss this version of the equivariant orbifold index formula. When $H$ acts trivially on $\mathfrak{X}$, we have

$$
K_{\text {orb }, H}^{0}(\mathfrak{X}) \cong K_{\text {orb }}^{0}(\mathfrak{X}) \otimes R(H),
$$

where $R(H)$ is the representation ring of $H$. Composing this isomorphism with the delocalized Chern character, we get

$$
\operatorname{ch}_{\text {deloc }, H}: K_{\text {orb }, H}^{0}(\mathfrak{X}) \longrightarrow H^{e v}(\tilde{\mathfrak{X}}, \mathbb{C}) \otimes R(H) .
$$


Then the equivariant orbifold index formula can be written as

$$
\operatorname{Index}_{H}\left(\not D^{+}\right)=\int_{\tilde{\mathfrak{X}}}^{o r b} \operatorname{ch} h_{\text {deloc }, H}(E) T d_{\text {deloc }}(\mathfrak{X}) .
$$

In particular, for each $h \in H$ and $E=\bigoplus_{i} E_{i} \otimes V_{i}$ under the isomorphism (2.3), we have

$$
\operatorname{Tr}\left(\left.h\right|_{K e r \not D^{+}}\right)-\operatorname{Tr}\left(\left.h\right|_{K e r \not D^{-}}\right)=\sum_{i} \operatorname{Index}\left(\not E_{E_{i}}^{+}\right) \operatorname{Tr}\left(\left.g\right|_{V_{i}}\right) .
$$

\section{INTRINSIC DESCRIPTION OF CHEN-RUAN COHOMOLOGY}

For an almost complex abelian orbifold $\mathfrak{X}$, Chen and Hu gave a classical definition of the Chen-Ruan product using an intrinsic definition of Chen-Ruan's obstruction bundle; see the proof of Proposition 1 in section 3.4 of 13 . For a smooth Deligne-Mumford stack, a similar description of Chen-Ruan's obstruction bundle was obtained by Jarvis, Kaufmann and Kimura in 24. In this section, we give an intrinsic definition of Chen-Ruan's obstruction bundle for an almost complex orbifold $\mathfrak{X}$ with associated Lie groupoid $\mathcal{G}$.

Let $\tilde{\mathfrak{X}}=\bigsqcup_{(g) \in \mathcal{T}_{1}} \mathfrak{X}_{(g)}$ be the inertia orbifold with associated inertia groupoid $\tilde{\mathcal{G}}$ and the evaluation map $e: \tilde{\mathcal{G}} \rightarrow \mathcal{G}$. The k-sector $\tilde{\mathfrak{X}}^{[k]}$ of $\mathfrak{X}$ is defined to be the orbifold on the set of all pairs

$$
\left(x,\left(g_{1}, \cdots, g_{k}\right)_{G_{x}}\right),
$$

where $\left(g_{1}, \cdots, g_{k}\right)_{G_{x}}$ denotes the conjugacy class of k-tuples. Here two k-tuples $\left(g_{1}^{(i)}, \cdots, g_{k}^{(i)}\right)_{G_{x}}, i=1,2$, are conjugate if there is a $g \in G_{x}$ such that $g_{j}^{(2)}=$ $g g_{j}^{(1)} g^{-1}$ for all $j=1, \cdots, k$. The k-sector orbifold $\tilde{\mathfrak{X}}^{[k]}$ consists of a disjoint union of sub-orbifolds of $\mathfrak{X}$,

$$
\tilde{\mathfrak{X}}^{[k]}=\bigsqcup_{(\mathbf{g}) \in \mathcal{T}_{k}} \mathfrak{X}_{(\mathbf{g})},
$$

where $\mathcal{T}_{k}$ denotes the set of equivalence classes of conjugacy k-tuples in local groups. Then the groupoid $\tilde{\mathcal{G}}^{[k]}$ associated to the k-sector $\tilde{\mathfrak{X}}^{[k]}$ for $k \geq 2$ is given by

$$
\tilde{\mathcal{G}}_{0}^{[k]}=\left\{\left(g_{1}, g_{2}, \cdots, g_{k}\right) \in \tilde{\mathcal{G}}_{0} \times_{e} \tilde{\mathcal{G}}_{0} \cdots \times_{e} \tilde{\mathcal{G}}_{0}\right\}
$$

and

$$
\tilde{\mathcal{G}}_{1}^{[k]}=\left\{\left(g_{1}, g_{2}, \cdots, g_{k}, h\right) \in\left(\tilde{\mathcal{G}}_{0}^{[k]}, \mathcal{G}_{1}\right) \mid t\left(g_{i}\right)=s(h)\right\},
$$

with the source map $s\left(g_{1}, g_{2}, \cdots, g_{k}, h\right)=\left(g_{1}, g_{2}, \cdots, g_{k}\right)$ and the target map $t\left(g_{1}, g_{2}, \cdots, g_{k}, h\right)=\left(h^{-1} g_{1} h, h^{-1} g_{2} h, \cdots, h^{-1} g_{k} h\right)$.

As in [14, $\tilde{\mathcal{G}}^{[k]}$ can be identified with the orbifold moduli space of constant pseudo-holomorphic maps from an orbifold sphere with $k+1$ orbifold points to $\mathfrak{X}$. The obstruction bundle over the orbifold moduli space, given by the cokernel of the Cauchy-Riemann operator over the orbifold sphere coupled with the pull-back of the tangent bundle $T \mathcal{G}$ of $\mathcal{G}$, defines a complex vector bundle $E^{[k]}$ over the groupoid of k-sectors $\tilde{\mathcal{G}}^{[k]}$, for $k \geq 2$.

Definition 3.1. Let $E$ be any complex vector bundle with an automorphism $\Phi$ of finite order over a proper étale groupoid $\mathcal{G}$. Choose a Hermitian metric on $E$ 
preserved by $\Phi$. Then $E$ has an eigen-bundle decomposition

$$
E=\bigoplus_{m_{i} \in \mathbb{Q} \cap[0,1)} E\left(m_{i}\right)
$$

where $\Phi$ acts on $E\left(m_{i}\right)$ as multiplication by $e^{2 \pi \sqrt{-1} m_{i}}$ for $m_{i} \in \mathbb{Q} \cap[0,1)$. We define

$$
E_{\Phi}=\sum_{m_{i} \in \mathbb{Q} \cap(0,1)} m_{i} E\left(m_{i}\right) \quad \text { and } \quad E_{\Phi^{-1}}=\sum_{m_{i} \in \mathbb{Q} \cap(0,1)}\left(1-m_{i}\right) E\left(m_{i}\right)
$$

as a linear combination of vector bundles with rational coefficients or as an element in $K^{0}(\mathcal{G}) \otimes \mathbb{Q}$.

Given an almost complex orbifold $\mathfrak{X}$, any orbifold complex vector bundle over a compact orbifold $\tilde{\mathfrak{X}}$, or equivalently any vector bundle over $\tilde{\mathcal{G}}$, has an automorphism. Specifically, if $E$ is a vector bundle over $\tilde{\mathcal{G}}$, then the fiber $E_{g}$ at $g \in \tilde{\mathcal{G}}_{0}$ has a linear isomorphism induced by the action of $g$. In particular, $e^{*} T \mathcal{G}$ is a complex vector bundle over $\tilde{\mathcal{G}}$ with an automorphism $\Phi$, and $T \tilde{\mathcal{G}}$ is a sub-bundle of $e^{*} T \mathcal{G}$ on which $\Phi$ acts trivially. Let $\mathcal{N}_{e}$ be the normal bundle of the evaluation map $e$ with the induced automorphism $\Phi$. We can choose a $\mathcal{G}$-invariant Hermitian metric on $\mathcal{G}$ such that $\Phi$ acts unitarily on $\mathcal{N}_{e}$. So the automorphism preserves the orthogonal decomposition

$$
e^{*} T \mathcal{G}=T \tilde{\mathcal{G}} \oplus \mathcal{N}_{e} .
$$

As a complex vector bundle over $\tilde{\mathcal{G}}, \mathcal{N}_{e}$ admits an eigen-bundle decomposition

$$
\mathcal{N}_{e}=\bigsqcup_{(g)} \mathcal{N}_{(g)}=\bigsqcup_{(g)} \bigoplus_{\theta_{(g)}} \mathcal{N}\left(\theta_{(g)}\right)
$$

where $\Phi$ acts on $\mathcal{N}\left(\theta_{(g)}\right)$ as multiplication by $e^{2 \pi \sqrt{-1} \theta_{(g)}}$ with $\theta_{(g)} \in(0,1)$, as $\Phi$ does not have eigenvalue 1 on $\mathcal{N}_{e}$.

Consider the following commutative diagram:

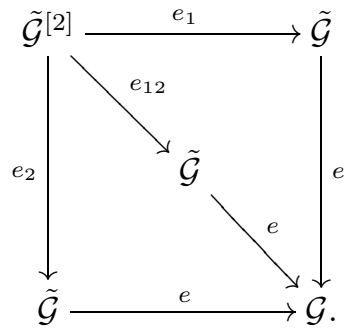

Let $\mathcal{N}$ be the normal bundle to the map $e \circ e_{12}=e \circ e_{1}=e \circ e_{2}: \tilde{\mathcal{G}}^{[2]} \rightarrow \mathcal{G}$. Then $\mathcal{N}$, as a complex vector bundle over $\tilde{\mathcal{G}}^{[2]}$, has three automorphisms:

(1) $\mathcal{N} \cong \mathcal{N}_{e_{1}} \oplus e_{1}^{*} \mathcal{N}_{e}$ with an automorphism $\Phi_{1}=I d \oplus e_{1}^{*} \Phi$; here $\mathcal{N}_{e_{1}}$ is the normal bundle to the map $e_{1}$. Then $\mathcal{N}_{\Phi_{1}}$, by Definition 3.1] is given by

$$
\mathcal{N}_{\Phi_{1}}=e_{1}^{*} \mathcal{N}_{e, \Phi}
$$

(2) $\mathcal{N} \cong \mathcal{N}_{e_{2}} \oplus e_{2}^{*} \mathcal{N}_{e}$ with an automorphism $\Phi_{2}=I d \oplus e_{2}^{*} \Phi$; here $\mathcal{N}_{e_{2}}$ is the normal bundle to the map $e_{2}$. Then we have

$$
\mathcal{N}_{\Phi_{2}}=e_{2}^{*} \mathcal{N}_{e, \Phi}
$$


(3) $\mathcal{N} \cong \mathcal{N}_{e_{12}} \oplus e_{12}^{*} \mathcal{N}_{e}$ with an automorphism $\Phi_{12}=I d \oplus e_{12}^{*} \Phi$; here $\mathcal{N}_{e_{12}}$ is the normal bundle to the map $e_{12}$. Then we have

$$
\mathcal{N}_{\Phi_{12}^{-1}}=e_{12}^{*} \mathcal{N}_{e, \Phi^{-1}} .
$$

When $\mathfrak{X}$ is compact, all of these three automorphisms have finite order.

The main result of this section is the following intrinsic description of the obstruction bundle $E^{[2]} \rightarrow \tilde{\mathcal{G}}^{[2]}$ in the definition of Chen-Ruan cohomology. This generalises the results of 13 and 24 to general almost complex orbifolds. Our proof using the orbifold index theorem was inspired by the proof in [13] for abelian cases, and it is simpler than the proof given in [24] for smooth Deligne-Mumford stacks.

Theorem 3.2. The obstruction bundle $E^{[2]}$ in the construction of the Chen-Ruan product satisfies the following identity:

$$
E^{[2]} \oplus \mathcal{N}=e_{1}^{*} \mathcal{N}_{e, \Phi}+e_{2}^{*} \mathcal{N}_{e, \Phi}+e_{12}^{*} \mathcal{N}_{e, \Phi^{-1}}
$$

in $K^{0}\left(\tilde{\mathcal{G}}^{[2]}\right) \otimes \mathbb{Q}$.

Proof. We first recall the definition of the obstruction bundle from Chapter 4.3 in [2] and Section 4 in [14. Identify $\tilde{\mathcal{G}}^{[2]}$ as the moduli space of constant representable orbifold morphisms from the orbifold Riemann sphere $S^{2}$ with three orbifold points to $\mathfrak{X}$. Given a point

$$
\left(g_{1}, g_{2}\right) \in \tilde{\mathcal{G}}_{0}^{[2]}
$$

with $s\left(g_{i}\right)=t\left(g_{i}\right)=x$, let $N$ be a finite subgroup generated by $g_{1}, g_{2}$ in the local group

$$
G_{x}=s^{-1}(x) \cap t^{-1}(x) .
$$

By Lemma 4.5 in 2, up to an isomorphism, $N$ depends only on the connected component of $\tilde{\mathfrak{X}}^{[2]}$, the orbifold associated to $\tilde{\mathcal{G}}^{[2]}$. Let $m_{1}, m_{2}$ and $m_{3}$ be the order of $g_{1}, g_{2}$ and $g_{1} g_{2}$ respectively. There is a smooth compact Riemann surface $\Sigma$ such that $\Sigma / N$ is an orbifold Riemann sphere $\left(S^{2},\left(m_{1}, m_{2}, m_{3}\right)\right)$ with three orbifold points of multiplicities $\left(m_{1}, m_{2}, m_{3}\right)$. Note that $\Sigma$ is given by the quotient of the orbifold universal cover of $\left(S^{2},\left(m_{1}, m_{2}, m_{3}\right)\right)$ by the kernel of the surjective homomorphism (cf. (4.14) in [2])

$$
\pi_{1}^{\text {orb }}\left(S^{2},\left(m_{1}, m_{2}, m_{3}\right)\right) \rightarrow N .
$$

The constant orbifold morphism corresponding to $\left(x, g_{1}, g_{2}\right)$ is represented by an ordinary constant map

$$
\tilde{f}_{x}: \Sigma \rightarrow \tilde{U}_{x}
$$

for an orbifold chart $\left(\tilde{U}_{x}, G_{x}\right)$ at $x$. The elliptic complex for the obstruction bundle over the point $\left(x, g_{1}, g_{2}\right)$ is the $N$-invariant part of the elliptic complex,

$$
\bar{\partial}_{\Sigma}: \Omega^{0}\left(\Sigma, T_{x} \mathcal{G}\right) \longrightarrow \Omega^{0,1}\left(\Sigma, T_{x} \mathcal{G}\right) .
$$

So the tangent space of the moduli space $\tilde{\mathcal{G}}^{[2]}$ at $\left(g_{1}, g_{2}\right) \in \tilde{\mathcal{G}}_{0}^{[2]}$ is given by

$$
T_{\left(g_{1}, g_{2}\right)} \tilde{\mathcal{G}}^{[2]}=\left(H^{0}(\Sigma) \otimes T_{x} \mathcal{G}\right)^{N}
$$

and the obstruction bundle over the point $\left(g_{1}, g_{2}\right) \in \tilde{\mathcal{G}}_{0}^{[2]}$ is given by

$$
E_{\left(g_{1}, g_{2}\right)}^{[2]}=\left(H^{0,1}(\Sigma) \otimes T_{x} \mathcal{G}\right)^{N},
$$


as $Z_{G_{x}}\left(g_{1}\right) \cap Z_{G_{x}}\left(g_{2}\right)$-representations. Here $Z_{G_{x}}\left(g_{1}\right)$ and $Z_{G_{x}}\left(g_{2}\right)$ are the centralizers of $g_{1}$ and $g_{2}$ respectively in the local group $G_{x}$.

Note that $\left(e \circ e_{12}\right)^{*} T_{x} \mathcal{G}=\mathcal{N}_{\left(g_{1}, g_{2}\right)} \oplus T_{\left(g_{1}, g_{2}\right)} \tilde{\mathcal{G}}^{[2]}$ and $N$ acts on $T_{\left(g_{1}, g_{2}\right)} \tilde{\mathcal{G}}^{[2]}$ trivially. We have

$$
\begin{aligned}
& \left(H^{0}(\Sigma) \otimes T_{\left(g_{1}, g_{2}\right)} \mathcal{G}\right)^{N} \\
= & \left(H^{0}(\Sigma) \otimes T_{\left(g_{1}, g_{2}\right)} \tilde{\mathcal{G}}^{[2]}\right)^{N} \oplus\left(H^{0}(\Sigma) \otimes \mathcal{N}_{\left(g_{1}, g_{2}\right)}\right)^{N} \\
= & T_{\left(g_{1}, g_{2}\right)} \tilde{\mathcal{G}}^{[2]} \oplus\left(H^{0}(\Sigma) \otimes \mathcal{N}_{\left(g_{1}, g_{2}\right)}\right)^{N} .
\end{aligned}
$$

Combining with (3.2), this leads to

$$
\left(H^{0}(\Sigma) \otimes \mathcal{N}_{\left(g_{1}, g_{2}\right)}\right)^{N}=0 .
$$

Applying the orbifold index formula (Proposition 4.2.2 of [14]) to the $N$-invariant part of the elliptic complex

$$
\left.\bar{\partial}_{\Sigma}\right|_{T_{\left(g_{1}, g_{2}\right)} \tilde{\mathcal{G}}^{[2]}}: \Omega^{0}\left(\Sigma, T_{\left(g_{1}, g_{2}\right)} \tilde{\mathcal{G}}^{[2]}\right) \longrightarrow \Omega^{0,1}\left(\Sigma, T_{\left(g_{1}, g_{2}\right)} \tilde{\mathcal{G}}^{[2]}\right)
$$

we get

$$
\operatorname{Index}\left(\left.\bar{\partial}_{\Sigma}\right|_{T_{\left(g_{1}, g_{2}\right)} \tilde{\mathcal{G}}^{[2]}}\right)=\operatorname{dim} T_{\left(g_{1}, g_{2}\right)} \tilde{\mathcal{G}}^{[2]}-0 .
$$

Here the contributions to the orbifold index formula from the three singular points are all zero due to the trivial action of $N$ on $T_{\left(g_{1}, g_{2}\right)} \tilde{\mathcal{G}}^{[2]}$. As

$$
\begin{aligned}
& \operatorname{Index}\left(\left.\bar{\partial}_{\Sigma}\right|_{\left.T_{\left(g_{1}, g_{2}\right)} \tilde{\mathcal{G}}^{[2]}\right)} \tilde{\mathcal{G}}^{[2]}\right)^{N}-\operatorname{dim}\left(H^{0,1}(\Sigma) \otimes T_{\left(g_{1}, g_{2}\right)} \tilde{\mathcal{G}}^{[2]}\right)^{N} \\
= & \operatorname{dim}\left(H^{0}(\Sigma) \otimes T_{\left(g_{1}, g_{2}\right.} \tilde{\mathcal{G}} \tilde{\mathcal{G}}^{[2]}\right)^{N}, \\
= & \operatorname{dim} T_{\left(g_{1}, g_{2}\right)} \tilde{\mathcal{G}}^{[2]}-\operatorname{dim}\left(H^{0,1}(\Sigma) \otimes T_{\left(g_{1}, g_{2}\right.}\right.
\end{aligned}
$$

together with (3.4) , we obtain $\left(H^{0,1}(\Sigma) \otimes T_{\left(g_{1}, g_{2}\right)} \tilde{\mathcal{G}}^{[2]}\right)^{N}=0$. Therefore,

$$
\begin{aligned}
E_{\left(g_{1}, g_{2}\right)}^{[2]} & =\left(H^{0,1}(\Sigma) \otimes T_{\left(g_{1}, g_{2}\right)} \tilde{\mathcal{G}}^{[2]}\right)^{N} \oplus\left(H^{0,1}(\Sigma) \otimes \mathcal{N}_{\left(g_{1}, g_{2}\right)}\right)^{N} \\
& =\left(H^{0,1}(\Sigma) \otimes \mathcal{N}_{\left(g_{1}, g_{2}\right)}\right)^{N}
\end{aligned}
$$

as $Z_{G_{x}}\left(g_{1}\right) \cap Z_{G_{x}}\left(g_{2}\right)$-representations, which can be glued together using the groupoid action to form a complex vector bundle $E^{[2]}$ over the groupoid $\tilde{\mathcal{G}}^{[2]}$.

Let

$$
\mathcal{N}_{\left(g_{1}, g_{2}\right)}=\bigoplus_{\lambda} V_{\lambda}
$$

be the decomposition into irreducible $Z_{G_{x}}\left(g_{1}\right) \cap Z_{G_{x}}\left(g_{2}\right)$-representations.

Note that the automorphisms $\Phi_{1}, \Phi_{2}$ and $\Phi_{12}^{-1}$, induced by the action of $g_{1}$, $g_{2}$ and $\left(g_{1} g_{2}\right)^{-1}$ respectively preserve each irreducible component $V_{\lambda}$ in (3.5) and commute with the action of $N$. Any eigen-subspace of $V_{\lambda}$ of $\Phi_{1}, \Phi_{2}$ or $\Phi_{12}^{-1}$ is also a representation of $Z_{G_{x}}\left(g_{1}\right) \cap Z_{G_{x}}\left(g_{2}\right)$. The irreducibility of $V_{\lambda}$ implies that

(1) $\left(V_{\lambda}\right)_{\Phi_{1}}=\theta_{1}^{\lambda} V_{\lambda}$, where $\Phi_{1}$ acts on $V_{\lambda}$ as multiplication by $e^{2 \pi \sqrt{-1} \theta_{1}^{\lambda}}$ for $\theta_{1} \in(0,1) \cap \mathbb{Q}$,

(2) $\left(V_{\lambda}\right)_{\Phi_{2}}=\theta_{2}^{\lambda} V_{\lambda}$, where $\Phi_{2}$ acts on $V_{\lambda}$ as multiplication by $e^{2 \pi \sqrt{-1} \theta_{2}^{\lambda}}$ for $\theta_{2} \in(0,1) \cap \mathbb{Q}$,

(3) $\left(V_{\lambda}\right)_{\Phi_{12}^{-1}}=\theta_{12}^{\lambda} V_{\lambda}$, where $\Phi_{12}^{-1}$ acts on $V_{\lambda}$ as multiplication by $e^{2 \pi \sqrt{-1} \theta_{12}^{\lambda}}$ for $\theta_{12} \in(0,1) \cap \mathbb{Q}$. 
Note $Z_{G_{x}}\left(g_{1}\right) \cap Z_{G_{x}}\left(g_{2}\right)$ acts trivially on the orbifold sphere $\left(S^{2},\left(m_{1}, m_{2}, m_{3}\right)\right)$. Applying the equivariant version of Kawasaki's orbifold index formula (see Remark 2.8) to the $N$-invariant part of the elliptic complex

$$
\bar{\partial}_{\Sigma}: \Omega^{0}\left(\Sigma, V_{\lambda}\right) \longrightarrow \Omega^{0,1}\left(\Sigma, V_{\lambda}\right),
$$

we get, for $g \in Z_{G_{x}}\left(g_{1}\right) \cap Z_{G_{x}}\left(g_{2}\right)$,

$$
\begin{aligned}
& \operatorname{Tr}\left(\left.g\right|_{\left(H^{0}(\Sigma) \otimes V_{\lambda}\right)^{N}}\right)-\operatorname{Tr}\left(\left.g\right|_{\left(H^{0,1}(\Sigma) \otimes V_{\lambda}\right)^{N}}\right) \\
= & \operatorname{Tr}\left(\left.g\right|_{V_{\lambda}}\right)-\left(\theta_{1}^{\lambda}+\theta_{2}^{\lambda}+\theta_{12}^{\lambda}\right) \operatorname{Tr}\left(\left.g\right|_{V_{\lambda}}\right) .
\end{aligned}
$$

From (3.3), we get $\left(H^{0}(\Sigma) \otimes V_{\lambda}\right)^{N}=0$ for each irreducible representation $V_{\lambda}$ in (3.5). Then (3.6) gives rise to

$$
\operatorname{Tr}\left(\left.g\right|_{\left(H^{0,1}(\Sigma) \otimes V_{\lambda}\right)^{N}}\right)+\operatorname{Tr}\left(\left.g\right|_{V_{\lambda}}\right)=\left(\theta_{1}^{\lambda}+\theta_{2}^{\lambda}+\theta_{12}^{\lambda}\right) \operatorname{Tr}\left(\left.g\right|_{V_{\lambda}}\right),
$$

for each $g \in Z_{G_{x}}\left(g_{1}\right) \cap Z_{G_{x}}\left(g_{2}\right)$. This implies that, as a representation of $Z_{G_{x}}\left(g_{1}\right) \cap$ $Z_{G_{x}}\left(g_{2}\right)$,

$$
\left(H^{0,1}(\Sigma) \otimes V_{\lambda}\right)^{N} \oplus V_{\lambda}=\left(V_{\lambda}\right)_{\Phi_{1}}+\left(V_{\lambda}\right)_{\Phi_{2}}+\left(V_{\lambda}\right)_{\Phi_{12}^{-1}}
$$

after combining like terms. Taking the direct sum over $\lambda$ as in (3.5), we obtain

$$
E_{\left(g_{1}, g_{2}\right)}^{[2]} \oplus \mathcal{N}=\left(e_{1}^{*} \mathcal{N}_{e, \Phi}\right)_{\left(g_{1}, g_{2}\right)}+\left(e_{2}^{*} \mathcal{N}_{e, \Phi}\right)_{\left(g_{1}, g_{2}\right)}+\left(e_{12}^{*} \mathcal{N}_{e, \Phi^{-1}}\right)_{\left(g_{1}, g_{2}\right)}
$$

in the representation ring of $Z_{G_{x}}\left(g_{1}\right) \cap Z_{G_{x}}\left(g_{2}\right)$ over the rational coefficients $\mathbb{Q}$.

The normal bundle $\mathcal{N}$ is a complex vector bundle over the groupoid $\tilde{\mathcal{G}}^{[2]}$, so the fiberwise identity (3.8) leads to the identity

$$
E^{[2]} \oplus \mathcal{N}=e_{1}^{*} \mathcal{N}_{e, \Phi}+e_{2}^{*} \mathcal{N}_{e, \Phi}+e_{12}^{*} \mathcal{N}_{e, \Phi^{-1}}
$$

in $K^{0}\left(\tilde{\mathcal{G}}^{[2]}\right) \otimes \mathbb{Q}$. This completes the proof of the theorem.

Remark 3.3. (1) One particular consequence of Theorem 3.2 is that the linear combination of vector bundles with rational coefficients

$$
e_{1}^{*} \mathcal{N}_{e, \Phi}+e_{2}^{*} \mathcal{N}_{e, \Phi}+e_{12}^{*} \mathcal{N}_{e, \Phi^{-1}}-\mathcal{N},
$$

after combining like terms, is a complex vector bundle which can be identified with the obstruction bundle $E^{[2]}$ in the construction of the Chen-Ruan product.

(2) In [22, Hepworth also provides another description of the obstruction bundle. As remarked in the introduction of [22, his description of the obstruction bundle involves the theory of orbifold Riemann surfaces to get certain inequalities regarding the degree shifting in the definition of the Chen-Ruan cohomology.

With this intrinsic description of the obstruction bundle, we can extend ChenHu's alternative definition of the Chen-Ruan cohomology ring for abelian orbifolds to general orbifolds by identifying

$$
H_{C R}^{*}(\mathfrak{X})=H^{*-2 \iota}(\tilde{\mathfrak{X}})
$$

with certain formal cohomology classes on $\mathfrak{X}$. We first recall the definition of the Chen-Ruan cohomology of an almost complex, compact orbifold. More details can be found in [14]. 
Definition 3.4. The Chen-Ruan cohomology $\left(H_{C R}^{*}(\mathfrak{X}), *_{C R}\right)$ of $\mathfrak{X}$ is defined to be

$$
H_{C R}^{d}(\mathfrak{X})=\bigoplus_{(g) \in \mathcal{T}_{1}} H^{d-2 \iota(g)}\left(\mathfrak{X}_{(g)}, \mathbb{C}\right)
$$

with a degree shift and a new product $*_{C R}$ given by the following formula. For $\omega_{1}, \omega_{2} \in H^{*}(\tilde{\mathfrak{X}}, \mathbb{C})$,

$$
\omega_{1} *_{C R} \omega_{2}=\left(e_{12}\right)_{*}\left(e_{1}^{*} \omega_{1} \cup e_{2}^{*} \omega_{2} \cup e\left(E^{[2]}\right)\right),
$$

where $e\left(E^{[2]}\right) \in H^{*}\left(\tilde{\mathfrak{X}}^{[2]}, \mathbb{C}\right)$ is the cohomological Euler class of the obstruction bundle $E^{[2]}$. The degree shifting number $\iota: \bigsqcup_{(g) \in \mathcal{T}_{1}} \mathfrak{X}_{(g)} \rightarrow \mathbb{Q}$ is defined to be

$$
\iota_{(g)}=\sum_{\theta_{(g)}} \operatorname{rank}_{\mathbb{C}}\left(\mathcal{N}\left(\theta_{(g)}\right)\right) \theta_{(g)},
$$

a locally constant function on $\tilde{\mathfrak{X}}$.

Remark 3.5. The Chen-Ruan product can also be defined using the 3-point function as follows. Given $\left(g_{1}, g_{2}\right) \in \mathcal{T}_{2}$, let $e_{1}, e_{2}$ and $e_{12}$ be the orbifold embeddings in (3.1). Denote

$$
e_{3}=I \circ e_{12}: \mathfrak{X}_{\left(g_{1}, g_{2}\right)} \longrightarrow \mathfrak{X}_{\left(g_{3}\right)},
$$

where $g_{3}=\left(g_{1} g_{2}\right)^{-1}$ and $I: \tilde{\mathfrak{X}} \rightarrow \tilde{\mathfrak{X}}$ is the involution defined by $\left(x,(g)_{G_{x}}\right) \mapsto$ $\left(x,\left(g^{-1}\right)_{G_{x}}\right)$. Then for $\omega_{1} \in H^{*}\left(\mathfrak{X}_{\left(g_{1}\right)}, \mathbb{C}\right)$ and $\omega_{2} \in H^{*}\left(\mathfrak{X}_{\left(g_{2}\right)}, \mathbb{C}\right)$, the Chen-Ruan product

$$
\omega_{1} *_{C R} \omega_{2} \in H^{*}\left(\mathfrak{X}_{\left(g_{1} g_{2}\right)}, \mathbb{C}\right)
$$

is uniquely determined by the 3 -point function

$$
\left\langle\omega_{1} *_{C R} \omega_{2}, \omega_{3}\right\rangle=\int_{\mathfrak{X}_{\left(g_{1}, g_{2}\right)}}^{\text {orb }} e_{1}^{*} \omega_{1} \wedge e_{2}^{*} \omega_{2} \wedge e_{3}^{*} \omega_{3} \wedge e\left(E_{\left(g_{1}, g_{1}\right)}^{[2]}\right)
$$

for any compactly supported $\omega_{3} \in H^{*}\left(\mathfrak{X}_{\left(g_{3}\right)}, \mathbb{C}\right)$.

Given an oriented real orbifold vector bundle $V$ over $\mathfrak{X}$ or, equivalently, an oriented vector bundle $V$ over $\mathcal{G}$, there is a compactly supported differential form $\Theta_{V}$ of $V$ such that

$$
\int_{V}^{o r b} \alpha \wedge \Theta_{V}=\int_{\mathfrak{X}}^{o r b} i^{*} \alpha
$$

for any differential form $\alpha$ on $V$, and $i: \mathfrak{X} \rightarrow V$ is the inclusion map defined by the zero section. Such a differential form defines the Thom class $\Theta(V)$ of $V$.

Denote by

$$
\Theta\left(\mathcal{N}_{e}\right)=\left(\cdots, \Theta\left(\mathcal{N}_{e_{(g)}}\right), \cdots\right) \in \bigoplus_{(g) \in \mathcal{T}_{1}} H^{*}\left(\mathcal{N}_{(g)}\right)
$$

the Thom class of the normal bundle $\mathcal{N}_{e}$ over $\tilde{\mathfrak{X}}=\bigsqcup_{(g) \in \mathcal{T}_{1}} \mathfrak{X}_{(g)}$ for the evaluation map $e: \tilde{\mathfrak{X}} \rightarrow \mathfrak{X}$. Note that the degree of $\Theta\left(\mathcal{N}_{(g)}\right)$ is $2 n_{(g)}$, where $n_{(g)}$ is the complex codimension of $\mathfrak{X}_{(g)}$ in $\mathfrak{X}$. The homomorphism

$$
H^{*}(\tilde{\mathfrak{X}})=\bigoplus_{(g) \in \mathcal{T}_{1}} H^{*}\left(\mathfrak{X}_{(g)}\right) \longrightarrow \bigoplus_{(g) \in \mathcal{T}_{1}} H^{*+2 n_{(g)}}\left(\mathcal{N}_{(g)}\right),
$$

sending $\omega_{(g)} \in H^{*}\left(\tilde{\mathfrak{X}}_{(g)}\right)$ to $\pi_{(g)}^{*} \omega_{(g)} \wedge \Theta\left(\mathcal{N}_{(g)}\right)$, is the Thom isomorphism. Here $\pi_{(g)}: \mathcal{N}_{e_{(g)}} \rightarrow \mathfrak{X}_{(g)}$ is the bundle projection. Identifying a neighbourhood of the 
zero section in $\mathcal{N}_{\left.e_{(} g\right)}$ containing the support of $\Theta\left(\mathcal{N}_{\left.e_{(} g\right)}\right)$, with the orbifold neighbourhood of $\tilde{\mathfrak{X}}_{(g)}$ in $\mathfrak{X}$, we obtain the push-forward map

$$
e_{*}: H^{*}(\tilde{\mathfrak{X}})=\bigoplus_{(g) \in \mathcal{T}_{1}} H^{*}\left(\mathfrak{X}_{(g)}\right) \longrightarrow \bigoplus_{(g) \in \mathcal{T}_{1}} H^{*+2 n_{(g)}}(\mathfrak{X}) .
$$

As before, let

$$
\mathcal{N}_{e}=\bigsqcup_{(g) \in \mathcal{T}_{1}} \bigoplus_{\theta_{(g)}} \mathcal{N}\left(\theta_{(g)}\right) \longrightarrow \bigsqcup_{(g)} \mathfrak{X}_{(g)}
$$

be the eigen-bundle decomposition for the automorphism $\Phi$.

Definition 3.6. The fractional Thom class of the normal bundle $\left(\mathcal{N}_{(g)}, \Phi\right)$ over $\mathfrak{X}_{(g)}$ is defined by the formal wedge product

$$
\Theta\left(\mathcal{N}_{(g)}, \Phi\right)=\bigwedge_{\theta_{(g)}}\left(\Theta\left(\mathcal{N}\left(\theta_{(g)}\right)\right)\right)^{\theta_{(g)}},
$$

of formal degree $2 \iota_{(g)}=\sum_{\theta_{(g)}} \theta_{(g)}$, with compact support in a neighbourhood of $\mathfrak{X}_{(g)}$ in $\mathfrak{X}$.

For each $(g) \in \mathcal{T}_{1}$, denote by $H_{\Phi}^{*+2 \iota(g)}(\mathfrak{X})$ the set of formal wedge products

$$
H_{\Phi}^{*+2 \iota(g)}(\mathfrak{X})=\left\{\omega \wedge \Theta\left(\mathcal{N}_{(g)}, \Phi\right) \mid \omega \in H^{*}(\mathfrak{X})\right\} .
$$

Elements in $H_{\Phi}^{*+2 \iota_{(g)}}(\mathfrak{X})$, called formal cohomology classes, can be represented by formal products of closed differential forms on $\mathfrak{X}$ and a formal fraction of differential forms for the fractional Thom class. These formal differential forms and their formal cohomology classes have the wedge product obtained from the formal product. We use the convention that integration of a formal differential form over $\mathfrak{X}$ vanishes unless its formal degree agrees with the dimension of $\mathfrak{X}$.

The fractional Thom class $\Theta\left(\mathcal{N}_{e}, \Phi\right)$ can be employed to define a formal pushforward map

$$
e_{*}^{\Phi}: H^{*}(\tilde{\mathfrak{X}})=\bigoplus_{(g) \in \mathcal{T}_{1}} H^{*}\left(\mathfrak{X}_{(g)}\right) \longrightarrow \bigoplus_{(g) \in \mathcal{T}_{1}} H_{\Phi}^{*+2 \iota(g)}(\mathfrak{X})
$$

obtained from the Thom isomorphism (3.10), with the usual Thom class replaced by the corresponding fractional Thom class. It is clear from the definition that $e_{*}^{\Phi}$ is injective.

Similarly, we can define another formal push-forward map

$$
e_{*}^{\Phi^{-1}}: H^{*}(\tilde{\mathfrak{X}})=\bigoplus_{(g) \in \mathcal{T}_{1}} H^{*}\left(\mathfrak{X}_{(g)}\right) \longrightarrow \bigoplus_{(g) \in \mathcal{T}_{1}} H_{\Phi^{-1}}^{*+2 n_{(g)}-2 \iota(g)}(\mathfrak{X})
$$

with the fractional Thom class $\Theta\left(\mathcal{N}_{(g)}, \Phi\right)$ replaced by

$$
\Theta\left(\mathcal{N}_{(g)}, \Phi^{-1}\right)=\bigwedge_{\theta_{(g)}}\left(\Theta\left(\mathcal{N}\left(\theta_{(g)}\right)\right)\right)^{1-\theta_{(g)}} .
$$

The following lemma explains the role of these two formal push-forward maps.

Lemma 3.7. The Thom class of the normal bundle $\mathcal{N}_{(g)} \rightarrow \mathfrak{X}_{(g)}$ is given by $\Theta\left(\mathcal{N}_{(g)}, \Phi\right) \wedge \Theta\left(\mathcal{N}_{(g)}, \Phi^{-1}\right)$. Moreover, for $\alpha \in H^{*}\left(\mathfrak{X}_{(g)}\right)$ and $\beta \in H^{*}\left(\mathfrak{X}_{\left(g^{-1}\right)}\right)$, we have

$$
\int_{\mathfrak{X}}^{o r b} e_{*}^{\Phi}(\alpha) \wedge e_{*}^{\Phi^{-1}}\left(I^{*} \beta\right)=\int_{\mathfrak{X}_{(g)}}^{\text {orb }} \alpha \wedge I^{*} \beta .
$$


Proof. Note that $\mathcal{N}_{(g)}=\bigoplus_{\theta_{(g)}} \mathcal{N}\left(\theta_{(g)}\right)$, from which we get

$$
\Theta\left(\mathcal{N}_{(g)}, \Phi\right)=\bigwedge_{\theta_{(g)}}\left(\Theta\left(\mathcal{N}\left(\theta_{(g)}\right)\right)\right)^{\theta_{(g)}}, \quad \Theta\left(\mathcal{N}_{(g)}, \Phi^{-1}\right)=\bigwedge_{\theta_{(g)}}\left(\Theta\left(\mathcal{N}\left(\theta_{(g)}\right)\right)\right)^{1-\theta_{(g)}} .
$$

Hence, $\Theta\left(\mathcal{N}_{(g)}, \Phi\right) \wedge \Theta\left(\mathcal{N}_{(g)}, \Phi^{-1}\right)=\bigwedge_{\theta}\left(\Theta\left(\mathcal{N}_{e}\right)\right)=\Theta\left(\mathcal{N}_{e}\right)$. From the definition of orbifold integration, we have

$$
\begin{aligned}
& \int_{\mathfrak{X}}^{o r b} e_{*}^{\Phi}(\alpha) \wedge e_{*}^{\Phi^{-1}}\left(I^{*} \beta\right) \\
= & \int_{\mathcal{N}_{(g)}}^{o r b} \pi_{(g)}^{*} \alpha \wedge \pi_{(g)}^{*} I^{*} \beta \wedge \Theta\left(\mathcal{N}_{(g)}, \Phi\right) \wedge \Theta\left(\mathcal{N}_{(g)}, \Phi^{-1}\right) \\
= & \int_{\mathcal{N}_{(g)}}^{\text {orb }} \pi_{(g)}^{*} \alpha \wedge \pi_{(g)}^{*} I^{*} \beta \wedge \Theta\left(\mathcal{N}_{(g)}\right) \\
= & \int_{\mathfrak{X}_{(g)}}^{\text {orb }} \alpha \wedge I^{*} \beta .
\end{aligned}
$$

Here $\pi_{(g)}: \mathcal{N}_{e_{(g)}} \rightarrow \mathfrak{X}_{(g)}$ is the bundle projection and $\mathcal{N}_{e_{(g)}}$ is identified with a neighbourhood of $\mathfrak{X}_{(g)}$ in $\mathfrak{X}$.

Theorem 3.8. Under the formal push-forward map

$$
e_{*}^{\Phi}: H^{*}(\tilde{\mathfrak{X}}) \longrightarrow \bigoplus_{(g) \in \mathcal{T}_{1}} H_{\Phi}^{*+2 \iota(g)}(\mathfrak{X}),
$$

the Chen-Ruan product becomes the wedge product. That is, for $\alpha, \beta \in H^{*}(\tilde{\mathfrak{X}})$,

$$
e_{*}^{\Phi}\left(\alpha *_{C R} \beta\right)=e_{*}^{\Phi}(\alpha) \wedge e_{*}^{\Phi}(\beta) .
$$

Proof. From the definition of the wedge product, $e_{*}^{\Phi}(\alpha) \wedge e_{*}^{\Phi}(\beta)$ is supported near $\tilde{\mathfrak{X}}^{[2]}$ or near the 0 -section of the normal bundle $\mathcal{N}$ over $\tilde{\mathfrak{X}}^{[2]}$.

Given $\left(g_{1}, g_{2}\right) \in \mathcal{T}_{2}$, let $e_{1}, e_{2}$ and $e_{3}$ be the orbifold embeddings of $\mathfrak{X}_{\left(g_{1}, g_{2}\right)}$ in $\mathfrak{X}_{\left(g_{1}\right)}, \mathfrak{X}_{\left(g_{2}\right)}$ and $\mathfrak{X}_{\left(g_{3}\right)}$ respectively, where $g_{3}=\left(g_{1} g_{2}\right)^{-1}$. Let $\pi_{(g)}$ be the projection $\mathcal{N}_{(g)} \rightarrow \widetilde{\mathfrak{X}}_{(g)}$. From Theorem 3.2 , we know that the Thom class $\Theta\left(E_{\left(g_{1}, g_{2}\right)}^{[2]}\right)$ satisfies

$$
\Theta\left(E_{g_{1}}^{[2]}\right) \wedge \Theta(\mathcal{N})=\Theta\left(\mathcal{N}_{\left(g_{1}\right)}, \Phi\right) \wedge \Theta\left(\mathcal{N}_{\left(g_{2}\right)}, \Phi\right) \wedge \Theta\left(\mathcal{N}_{\left(g_{3}\right)}, \Phi\right)
$$

as a formal cohomology class supported near a neighbourhood of $\mathfrak{X}_{\left(g_{1}, g_{2}\right)}^{[2]}$ in $\mathfrak{X}$. For $\alpha \in H^{*}\left(\mathfrak{X}_{\left(g_{1}\right)}\right), \beta \in H^{*}\left(\mathfrak{X}_{\left(g_{2}\right)}\right)$ and $\gamma \in H_{c}^{*}\left(\mathfrak{X}_{\left(g_{3}\right)}\right)$, near $\mathfrak{X}_{\left(g_{1}, g_{2}\right)}$ we have

$$
\begin{aligned}
& \int_{\mathfrak{X}}^{\text {orb }} e_{*}^{\Phi}(\alpha) \wedge e_{*}^{\Phi}(\beta) \wedge e_{*}^{\Phi}(\gamma) \\
= & \int_{\mathfrak{X}}^{o r b} \pi_{\left(g_{1}\right)}^{*}(\alpha) \wedge \pi_{\left(g_{2}\right)}^{*}(\beta) \wedge \pi_{\left(g_{3}\right)}^{*}(\gamma) \wedge \Theta\left(\mathcal{N}_{\left(g_{1}\right)}, \Phi\right) \wedge \Theta\left(\mathcal{N}_{\left(g_{2}\right)}, \Phi\right) \wedge \Theta\left(\mathcal{N}_{\left(g_{3}\right)}, \Phi\right) \\
= & \int_{\mathfrak{X}}^{o r b} \pi_{\left(g_{1}\right)}^{*}(\alpha) \wedge \pi_{\left(g_{2}\right)}^{*}(\beta) \wedge \pi_{\left(g_{3}\right)}^{*}(\gamma) \wedge \Theta\left(E_{\left(g_{1}, g_{2}\right)}^{[2]}\right) \wedge \Theta(\mathcal{N}) \\
= & \int_{\mathfrak{X}_{\left(g_{1}, g_{2}\right)}}^{\text {orb }} e_{1}^{*}(\alpha) \wedge e_{2}^{*}(\beta) \wedge e_{3}^{*}(\gamma) \wedge e\left(E_{\left(g_{1}, g_{2}\right)}^{[2]}\right) .
\end{aligned}
$$


By Poincáre duality for orbifolds and the 3-point function (3.9) for the Chen-Ruan product, we get

$$
e_{*}^{\Phi}\left(\alpha *_{C R} \beta\right)=e_{*}^{\Phi}(\alpha) \wedge e_{*}^{\Phi}(\beta) .
$$

\section{Stringy PRoduct on the ORBIFOLd K-THEORY}

In this section, we will define a stringy product on the orbifold K-theory of an almost compact orbifold $\mathfrak{X}$ and establish a ring isomorphism between the orbifold K-theory of $\mathfrak{X}$ and the Chen-Ruan cohomology ring using a modified version of the delocalized Chern character. We first recall the definition of the delocalized Chern character and its properties. Then we give a geometric definition of a stringy product on $K_{\text {orb }}^{*}(\mathfrak{X}) \otimes \mathbb{C}$ itself and show that this product agrees with the ChenRuan product under a modified version of the delocalized Chern character.

4.1. Review of the Adem-Ruan-Zhang stringy product. In [4, Adem, Ruan and Zhang defined a stringy product on the twisted K-theory of the inertia orbifold $\tilde{\mathfrak{X}}$ of a compact, almost complex orbifold $\mathfrak{X}$. This product will be called the AdemRuan-Zhang product, denoted by $\bullet A R Z$. To simplify the construction, we first discuss the untwisted case for a presentable, compact, almost complex orbifold $\mathfrak{X}$.

The Adem-Ruan-Zhang product on the orbifold K-theory of the inertia orbifold $\tilde{\mathfrak{X}}$, under the canonical isomorphism for a presentable orbifold $\mathfrak{X}$,

$$
K_{\text {orb }}^{*}(\tilde{\mathfrak{X}}) \cong K^{*}(\tilde{\mathcal{G}}),
$$

is given by the formula

$$
\alpha \bullet{ }_{A R Z} \beta=\left(e_{12}\right)_{*}\left(e_{1}^{*} \alpha \cdot e_{2}^{*} \beta \cdot \lambda_{-1}\left(E^{[2]}\right)\right),
$$

for $\alpha, \beta \in K^{*}(\tilde{\mathcal{G}})$, where $\lambda_{-1}\left(E^{[2]}\right)=\left[\Lambda_{\mathbb{C}}^{\text {even }} E^{[2]}\right]-\left[\Lambda_{\mathbb{C}}^{\text {odd }} E^{[2]}\right] \in K^{*}\left(\tilde{\mathcal{G}}^{[2]}\right)$ is the K-theoretical Euler class of the obstruction bundle $E^{[2]}$. We recall here that the push-forward map

$$
\left(e_{12}\right)_{*}: K^{*}\left(\tilde{\mathcal{G}}^{[2]}\right) \longrightarrow K^{*}(\tilde{\mathcal{G}})
$$

is given by the composition of the Thom isomorphism

$$
K^{*}\left(\tilde{\mathcal{G}}^{[2]}\right) \cong K_{c}^{*}\left(\mathcal{N}_{e_{12}}\right)
$$

and the natural extension homomorphism $K_{c}^{*}\left(\mathcal{N}_{e_{12}}\right) \rightarrow K^{*}(\tilde{\mathcal{G}})$ obtained by identifying the normal bundle $\mathcal{N}_{e_{12}}$ as a (component-wise) tubular neighbourhood of $\tilde{\mathcal{G}}^{[2]}$ in $\tilde{\mathcal{G}}$. Under the decomposition

$$
K_{\text {orb }}^{*}(\tilde{\mathfrak{X}})=\bigoplus_{(g)} K_{\text {orb }}^{*}\left(\mathfrak{X}_{(g)}\right),
$$

for $\alpha_{1} \in K_{\text {orb }}^{*}\left(\mathfrak{X}_{\left(g_{1}\right)}\right)$ and $\alpha_{2} \in K_{\text {orb }}^{*}\left(\mathfrak{X}_{\left(g_{2}\right)}\right)$ we have

$$
\alpha_{1} \bullet A R Z \alpha_{2} \in K_{\text {orb }}^{*}\left(\mathfrak{X}_{\left(g_{1} g_{2}\right)}\right) \text {. }
$$

For an abelian almost complex orbifold $\mathfrak{X}$ obtained from a compact action of an abelian Lie group on a compact manifold, Becerra and Uribe in [10] established a ring homomorphism

$$
\left(K_{\text {orb }}^{*}(\tilde{\mathfrak{X}}), \bullet A R Z\right) \longrightarrow\left(H_{d R}^{*}(\tilde{\mathfrak{X}}, \mathbb{C}), *_{C R}\right)
$$


by modifying the usual Chern character as in [24. The decomposition theorem of Adem-Ruan (Theorem 5.1 in [3]) implies that this ring homomorphism is not an isomorphism in general.

Now we briefly recall the definition of twisted K-theory for orbifolds. A twisting on an orbifold groupoid $\mathcal{G}$ is a generalized morphism

$$
\sigma: \mathcal{G} \longrightarrow P U(H)
$$

where $P U(H)$, viewed as a groupoid with the unit space $\{e\}$, is the projective unitary group $P U(H)$ of an infinite-dimensional separable complex Hilbert space $H$, with the norm topology. A twisting $\sigma$ gives rise to a principal $P U(H)$-bundle $\mathcal{P}_{\sigma}$ over $\mathcal{G}$. Two twistings are called equivalent if their associated $P U(H)$-bundles are isomorphic.

Remark 4.1. It was argued in [6] that it is not desirable to use the norm topology on the structure group $P U(H)$, particularly in dealing with equivariant twisted K-theory. For orbifolds and orbifold groupoids, it suffices to consider $P U(H)$ with the norm topology in the definition of twistings and twisted K-theory just as in the non-equivariant case. The main reason is that the underlying groupoid for any orbifold is proper and étale, so locally it is given by the transformation groupoid for a finite group action. As pointed out in [6], the structure group $P U(H)$ with the norm topology works fine for an almost free action of a compact Lie group, where the underlying orbifold is presentable.

Let $\mathcal{K}(H)$ be a space of compact operators on $H$ endowed with the norm topology, and let $\operatorname{Fred}(H)$ be the space of Fredholm operators endowed with the $*$-strong topology and Fred $^{1}(H)$ be the space of self-adjoint elements in Fred $(H)$. Consider the associated bundles

$$
\operatorname{Fred}^{i}\left(\mathcal{P}_{\sigma}\right)=\mathcal{P}_{\sigma} \times_{P U(H)} \operatorname{Fred}^{i}(H)
$$

over the groupoid $\mathcal{G}$. The $\sigma$-twisted K-theory of $\mathcal{G}$, denoted $K^{i}(\mathcal{G}, \sigma)$ for $i=0,1$, is defined to be the set of homotopy classes of compactly supported sections of $\operatorname{Fred}^{i}\left(\mathcal{P}_{\sigma}\right)$. For any orbifold $\mathfrak{X}=(X, \mathcal{U})$, the twisted K-theory of $\mathfrak{X}$ is defined to be the twisted K-theory of the associated proper étale groupoid $\mathcal{G}[\mathcal{U}]$. Then the twisted orbifold K-theory is a module over the ordinary orbifold K-theory and satisfies the Mayer-Vieroris sequence and the Thom isomorphism for complex orbifold vector bundles. Moreover, the twisted orbifold K-theory admits the multiplicative operation

$$
K_{\text {orb }}^{*}\left(\mathfrak{X}, \sigma_{1}\right) \otimes K_{\text {orb }}^{*}\left(\mathfrak{X}, \sigma_{2}\right) \longrightarrow K_{\text {orb }}^{*}\left(\mathfrak{X}, \sigma_{1}+\sigma_{2}\right),
$$

where the addition $\sigma_{1}+\sigma_{2}$ is the new twisting from the group homomorphism $P U(H) \times P U(H) \rightarrow P U(H)$ induced by the Hilbert space tensor product $H \cong$ $H \otimes H$. See [44, 27], 43] for more detailed discussions. by

Given a twisting $\tilde{\sigma}$ on $\tilde{\mathcal{G}}$, Adem, Ruan and Zhang defined a product on $K_{\text {orb }}^{*}(\mathfrak{X}, \tilde{\sigma})$

$$
\alpha \bullet A R Z \beta=\left(e_{12}\right)_{*}\left(e_{1}^{*} \alpha \cdot e_{2}^{*} \beta \cdot \lambda_{-1}\left(E^{[2]}\right)\right),
$$

for $\alpha, \beta \in K_{\text {orb }}^{*}(\tilde{\mathfrak{X}}, \tilde{\sigma})=K^{*}(\tilde{\mathcal{G}}, \tilde{\sigma})$. Here applying the multiplicative operation (4.3) and the $K_{\text {orb }}^{*}(\mathfrak{X})$-module structure,

$$
e_{1}^{*} \alpha \cdot e_{2}^{*} \beta \cdot \lambda_{-1}\left(E^{[2]}\right)
$$


is an element in $K_{\text {orb }}^{*}\left(\tilde{\mathcal{G}}^{[2]}, e_{1}^{*} \tilde{\sigma}+e_{2}^{*} \tilde{\sigma}\right)$, and

$$
\left(e_{12}\right)_{*}: K_{\text {orb }}^{*}\left(\tilde{\mathcal{G}}^{[2]}, e_{12}^{*} \tilde{\sigma}\right) \longrightarrow K_{\text {orb }}^{*}(\tilde{\mathcal{G}}, \tilde{\sigma})
$$

is the push-forward map for (component-wise) orbifold embeddings obtained by the composition of the Thom isomorphism in twisted K-theory and the natural extension homomorphism for open embeddings. To make sense of the expression (4.4), we need a canonical identification

$$
K_{\text {orb }}^{*}\left(\tilde{\mathcal{G}}^{[2]}, e_{1}^{*} \tilde{\sigma}+e_{2}^{*} \tilde{\sigma}\right) \cong K_{\text {orb }}^{*}\left(\tilde{\mathcal{G}}^{[2]}, e_{12}^{*} \tilde{\sigma}\right)
$$

which is guaranteed by the transgressive property of the twisting $\tilde{\sigma}$; see [4] for details.

4.2. Stringy product on orbifold K-theory. Let $\mathfrak{X}$ be an almost complex compact orbifold and $\tilde{\mathfrak{X}}=\bigsqcup_{(g)} \mathfrak{X}_{(g)}$ be its inertia orbifold. Let $\mathcal{G}$ and $\tilde{\mathcal{G}}$ be their presenting proper étale groupoids. The evaluation map $e=\bigsqcup_{(g)} e_{(g)}: \bigsqcup_{(g)} \mathfrak{X}_{(g)} \rightarrow \mathfrak{X}$ is presented by the map $e: \tilde{\mathcal{G}} \rightarrow \mathcal{G}$.

Proposition 4.2. There exists a canonical ring homomorphism

$$
c h_{\Phi}: K_{\text {orb }}^{*}(\tilde{\mathfrak{X}}) \longrightarrow H^{*}(\tilde{\mathfrak{X}}, \mathbb{C})
$$

such that the diagram

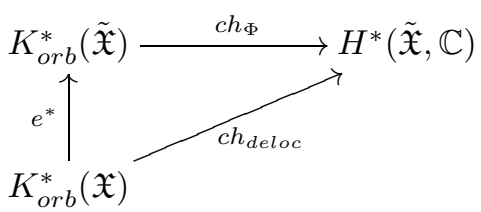

commutes.

Proof. Any complex orbifold vector bundle $\mathcal{E}$ over the inertia orbifold $\tilde{\mathfrak{X}}$ admits an automorphism $\Phi$. In terms of the associated complex vector bundle $E$ over the canonical proper étale groupoid $\tilde{\mathcal{G}}$, the action of $\Phi$ on each fiber $E_{g}$ over the point $g \in \tilde{\mathcal{G}}_{0}$ is given by the action of $g$. We have an eigen-bundle decomposition of $\mathcal{E}$

$$
\mathcal{E}=\bigoplus_{\theta \in \mathbb{Q} \cap[0,1)} \mathcal{E}_{\theta}
$$

where $\mathcal{E}_{\theta}$ is a complex vector bundle over $\tilde{\mathcal{G}}$, on which $\Phi$ acts as multiplication by $e^{2 \pi \sqrt{-1} \theta}$. Then

$$
\operatorname{ch}_{\Phi}(\mathcal{E})=\sum_{\theta} e^{2 \pi \sqrt{-1} \theta} \operatorname{ch}\left(\mathcal{E}_{\theta}\right) \in H^{e v}(\tilde{\mathcal{G}}, \mathbb{C}) \cong H^{e v}(\tilde{\mathfrak{X}}, \mathbb{C})
$$

defines a homomorphism

$$
c h_{\Phi}: K_{\text {orb }}^{0}(\tilde{\mathfrak{X}}) \longrightarrow H^{e v}(\tilde{\mathfrak{X}}, \mathbb{C}) .
$$

From the definition of the delocalized Chern character, we get $c h_{\text {deloc }}=c h_{\Phi} \circ e^{*}$. Hence, diagram (4.5) commutes. 
Proposition 4.2 implies the commutative diagram

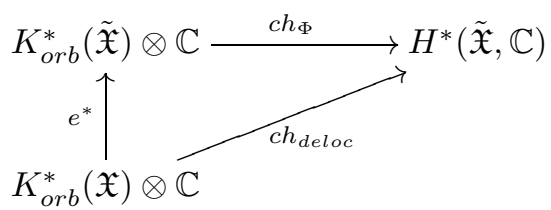

where $c_{\text {deloc }}$ is an isomorphism.

For any element $\tilde{\alpha} \in \operatorname{Im}\left(e^{*}\right) \cap \operatorname{Ker}\left(\operatorname{ch} h_{\Phi}\right)$, there exists $\alpha \in K_{\text {orb }}^{*}(\tilde{\mathfrak{X}}) \otimes \mathbb{C}$ such that $\tilde{\alpha}=e^{*}(\alpha)$ and $c h_{\Phi}(\tilde{\alpha})=0$. These imply that $c h_{\text {deloc }}(\alpha)=0$. Hence $\alpha=0$, as $c h_{d e l o c}$ is an isomorphism. Then $\tilde{\alpha}=e^{*}(\alpha)=e^{*}(0)=0$. Then we obtain

$$
K_{\text {orb }}^{*}(\tilde{\mathfrak{X}}) \otimes \mathbb{C} \cong \operatorname{Im}\left(e^{*}\right) \oplus \operatorname{Ker}(\operatorname{ch} \Phi) .
$$

Hence, each element $\tilde{\alpha} \in K_{\text {orb }}^{*}(\tilde{\mathfrak{X}}) \otimes_{\mathbb{Z}} \mathbb{C}$ can be uniquely written as

$$
\tilde{\alpha}=e^{*} \alpha+\beta
$$

for a unique element $\alpha \in K_{\text {orb }}^{*}(\mathfrak{X}) \otimes_{\mathbb{Z}} \mathbb{C}$ and $\beta \in \operatorname{Ker}\left(\operatorname{ch}_{\Phi}\right)$. Define

$$
e_{\#}: K_{\text {orb }}^{*}(\tilde{\mathfrak{X}}) \otimes_{\mathbb{Z}} \mathbb{C} \longrightarrow K_{\text {orb }}^{*}(\mathfrak{X}) \otimes_{\mathbb{Z}} \mathbb{C},
$$

sending $\tilde{\alpha}=e^{*} \alpha+\beta$ as in (4.8) to $\alpha$. Then one can check that $e_{\#}$ is a left inverse of $e^{*}$ such that

$$
\left(\operatorname{ch}_{\text {deloc }} \circ e_{\#}\right)(\tilde{\alpha})=\operatorname{ch}_{\Phi}(\tilde{\alpha})
$$

for any $\tilde{\alpha} \in K_{\text {orb }}^{*}(\tilde{\mathfrak{X}}) \otimes_{\mathbb{Z}} \mathbb{C}$.

With these preparations, we now can define the stringy product on the orbifold K-theory of a compact almost complex orbifold $\mathfrak{X}$. For simplicity, we will denote $K_{\text {orb }}^{*}(\mathfrak{X}) \otimes_{\mathbb{Z}} \mathbb{C}$ and $K_{\text {orb }}^{*}(\tilde{\mathfrak{X}}) \otimes_{\mathbb{Z}} \mathbb{C}$ by $K_{\text {orb }}^{*}(\mathfrak{X}, \mathbb{C})$ and $K_{\text {orb }}^{*}(\tilde{\mathfrak{X}}, \mathbb{C})$ respectively.

Definition 4.3. Let $\mathfrak{X}$ be an almost complex compact orbifold and $\tilde{\mathfrak{X}}=\bigsqcup_{(g)} \mathfrak{X}_{(g)}$ be its inertia orbifold. The stringy product $\circ$ on $K_{\text {orb }}^{*}(\mathfrak{X}, \mathbb{C})$ is defined by

$$
\alpha_{1} \circ \alpha_{2}=e_{\#}\left(e^{*} \alpha_{1} \bullet A R Z e^{*} \alpha_{2}\right)
$$

for $\alpha_{1}, \alpha_{2} \in K_{\text {orb }}^{*}(\mathfrak{X}, \mathbb{C})$. Here $e^{*} \alpha_{1} \bullet A R Z e^{*} \alpha_{2}$ is the Adem-Ruan-Zhang stringy product on $K_{\text {orb }}^{*}(\tilde{\mathfrak{X}}, \mathbb{C})$ and $e_{\#}$ is the left inverse of $e^{*}$.

Next, we define a modified version of the delocalized Chern character,

$$
\widetilde{c h}_{\text {deloc }}: K_{\text {orb }}^{*}(\mathfrak{X}) \longrightarrow H^{*}(\tilde{\mathfrak{X}}, \mathbb{C}) .
$$

For a complex vector bundle $\mathcal{E}$ over a compact manifold $M$, there is a well-known Chern character defect for Thom isomorphisms in K-theory and cohomology theory (for example see Chapter III.12 in [26]) such that the diagram

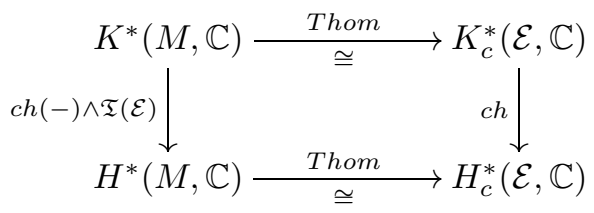

commutes. Here $\mathfrak{T}(\mathcal{E})$ is a characteristic class of $\mathcal{E}$,

$$
\mathfrak{T}(\mathcal{E})=\frac{\operatorname{ch}\left(\lambda_{-1}(\mathcal{E})\right)}{e(\mathcal{E})} \in H^{*}(M, \mathbb{C})
$$


associated to the formal power series

$$
\mathfrak{T}(x)=\frac{1-e^{x}}{x} .
$$

That is, if we formally split the total Chern class as $c(\mathcal{E})=\prod\left(1+x_{j}\right)$, then

$$
\mathfrak{T}(\mathcal{E})=\prod_{i} \frac{1-e^{x_{i}}}{x_{i}} .
$$

Given an orbifold complex vector bundle over the inertia orbifold $\tilde{\mathfrak{X}}$, let

$$
\mathcal{E}=\bigoplus_{m_{i} \in \mathbb{Q} \cap[0,1)} \mathcal{E}_{m_{i}}
$$

be the eigen-bundle decomposition of the canonical automorphism $\Phi$, where $\Phi$ acts on $\mathcal{E}_{m_{i}}$ as multiplication by $e^{2 \pi \sqrt{-1} m_{i}}$. Define a cohomology class

$$
\mathfrak{T}(\mathcal{E}, \Phi)=\prod_{m_{i}} \mathfrak{T}\left(\mathcal{E}_{m_{i}}\right)^{m_{i}} \in H^{*}(\tilde{\mathfrak{X}}, \mathbb{C}),
$$

where $\mathfrak{T}\left(\mathcal{E}_{m_{i}}\right)^{m_{i}}$ is the characteristic class associated to the formal power series $\left(\frac{1-e^{x}}{x}\right)^{m}$. Then $\mathfrak{T}(\mathcal{E}, \Phi)$ is an invertible element in $H^{*}(\tilde{\mathfrak{X}}, \mathbb{C})$, as the degree zero component is 1 .

Associated to the normal bundle

$$
\mathcal{N}_{e}=\bigsqcup_{(g) \in \mathcal{T}_{1}} \mathcal{N}_{(g)}
$$

over $\tilde{\mathfrak{X}}=\bigsqcup_{(g)} \mathfrak{X}_{(g)}$, we have the cohomology class $\mathfrak{T}\left(\mathcal{N}_{e}, \Phi\right)$ in $H^{*}(\tilde{\mathfrak{X}})$ whose component in $H^{*}\left(\mathfrak{X}_{(g)}, \mathbb{C}\right)$ is given by $\mathfrak{T}\left(\mathcal{N}_{(g)}, \Phi\right)$. The intrinsic description of the obstruction bundles over $\tilde{\mathfrak{X}}^{[2]}$ in Theorem 3.2 gives rise to the following identity:

$$
\begin{aligned}
& \mathfrak{T}\left(E_{\left(g_{1}, g_{2}\right)}^{2]} \oplus \mathcal{N}_{\left(g_{1}, g_{2}\right)}\right) \wedge e_{12}^{*} \mathfrak{T}\left(\mathcal{N}_{\left(g_{1} g_{2}\right)}, \Phi\right) \\
= & e_{1}^{*} \mathfrak{T}\left(\mathcal{N}_{\left(g_{1}\right)}, \Phi\right) \wedge e_{2}^{*} \mathfrak{T}\left(\mathcal{N}_{\left(g_{2}\right)}, \Phi\right)
\end{aligned}
$$

in $H^{*}\left(\mathfrak{X}_{\left(g_{1}, g_{2}\right)}, \mathbb{C}\right)$ for any connected component $\mathfrak{X}_{\left(g_{1}, g_{2}\right)}$ of $\tilde{\mathfrak{X}}_{[2]}$. Here $\mathcal{N}_{\left(g_{1}, g_{2}\right)}$ is the normal bundle for the orbifold embedding $e_{12}: \mathfrak{X}_{\left(g_{1}, g_{2}\right)} \rightarrow \mathfrak{X}_{\left(g_{1} g_{2}\right)}$.

Definition 4.4. The modified delocalized Chern character on the orbifold K-theory $K_{\text {orb }}^{*}(\mathfrak{X}) \cong K^{*}(\mathcal{G})$ is defined to be

$$
\widetilde{c h}_{\text {deloc }}=\mathfrak{T}\left(\mathcal{N}_{e}, \Phi\right) \wedge \text { ch deloc }: K_{\text {orb }}^{*}(\mathfrak{X}) \longrightarrow H^{*}(\tilde{\mathfrak{X}}, \mathbb{C}) .
$$

Now we can prove the main result of this paper.

Theorem 4.5. Let $\mathfrak{X}$ be a compact, almost complex, effective orbifold. The modified delocalized Chern character,

$$
\widetilde{c h}_{\text {deloc }}:\left(K_{\text {orb }}^{*}(\mathfrak{X}, \mathbb{C}), \circ\right) \longrightarrow\left(H^{*}(\tilde{\mathfrak{X}}, \mathbb{C}), *_{C R}\right),
$$

is a ring isomorphism between two $\mathbb{Z}_{2}$-graded multiplicative cohomology theories.

Proof. By the definition of stringy product (Definition 4.3) and (4.7)-(4.10), we get

$$
\begin{aligned}
& \operatorname{ch}_{\text {deloc }}\left(\alpha_{1} \circ \alpha_{2}\right) \\
= & \left(\operatorname{ch}_{\text {deloc }} \circ e_{\#}\right)\left(e^{*} \alpha_{1} \bullet \text { ARZ } e^{*} \alpha_{2}\right) \\
= & c h_{\Phi}\left(e^{*} \alpha_{1} \bullet \text { ARZ } e^{*} \alpha_{2}\right) \\
= & c h_{\Phi}\left[\left(e_{12}\right)_{*}\left(e_{1}^{*} e^{*} \alpha_{1} \cdot e_{2}^{*} e^{*} \alpha_{2} \cdot \lambda_{-1}\left(E^{[2]}\right)\right)\right]
\end{aligned}
$$


for $\alpha_{1}, \alpha_{2} \in K_{\text {orb }}^{*}(\mathfrak{X}, \mathbb{C})$. As $e^{*}=\bigoplus_{(g)} e_{(g)}^{*}$, the $\mathfrak{X}_{(g)}$-component of ch $_{\text {deloc }}\left(\alpha_{1} \circ \alpha_{2}\right)$ in (4.13) is given by

$$
\sum_{\left\{\left(g_{1}, g_{2}\right):\left(g_{1} g_{2}\right)=(g)\right\}} \operatorname{ch}_{\Phi}\left[\left(e_{12}\right)_{*}\left(e_{1}^{*} e_{\left(g_{1}\right)}^{*} \alpha_{1} \cdot e_{2}^{*} e_{\left(g_{2}\right)}^{*} \alpha_{2} \cdot \lambda_{-1}\left(E_{\left(g_{1}, g_{2}\right)}^{[2]}\right)\right)\right] .
$$

Here the pushforward map $\left(e_{12}\right)_{*}: K_{\text {orb }}\left(\mathfrak{X}_{\left(g_{1}, g_{2}\right)}, \mathbb{C}\right) \rightarrow K_{\text {orb }}\left(\mathfrak{X}_{\left(g_{1} g_{2}\right)}, \mathbb{C}\right)$ is obtained by the composition of the Thom isomorphism for the normal bundle $\mathcal{N}_{\left(g_{1}, g_{2}\right)}$ of $e_{12}: \mathfrak{X}_{\left(g_{1}, g_{2}\right)} \rightarrow \mathfrak{X}_{\left(g_{1} g_{2}\right)}$ and the natural extension for open embeddings. Using the fact that the automorphism on the Thom class of $\mathcal{N}_{\left(g_{1}, g_{2}\right)}$ is trivial, we obtain

$$
\begin{aligned}
& \widetilde{\operatorname{ch}}_{\text {deloc }}\left(\alpha_{1} \circ \alpha_{2}\right) \\
& =\operatorname{ch}_{\text {deloc }}\left(\alpha_{1} \circ \alpha_{2}\right) \wedge \mathfrak{T}\left(\mathcal{N}_{e}, \Phi\right) \\
& =\sum_{\left\{\left(g_{1}, g_{2}\right)\right\}} \operatorname{ch}_{\Phi}\left[\left(e_{12}\right)_{*}\left(e_{1}^{*} e_{\left(g_{1}\right)}^{*} \alpha_{1} \cdot e_{2}^{*} e_{\left(g_{2}\right)}^{*} \alpha_{2} \cdot \lambda_{-1}\left(E_{\left(g_{1}, g_{2}\right)}^{[2]}\right)\right)\right] \wedge \mathfrak{T}\left(\mathcal{N}_{\left(g_{1} g_{2}\right)}, \Phi\right) \\
& =\sum_{\left\{\left(g_{1}, g_{2}\right)\right\}}\left(e_{12}\right)_{*}\left[e_{1}^{*} c h_{\Phi}\left(e_{\left(g_{1}\right)}^{*} \alpha_{1}\right) \wedge e_{2}^{*} \operatorname{ch}_{\Phi}\left(e_{\left(g_{2}\right)}^{*} \alpha_{2}\right) \wedge \operatorname{ch}\left(\lambda_{-1}\left(E_{\left(g_{1}, g_{2}\right)}^{[2]}\right)\right)\right. \\
& \left.\wedge \mathfrak{T}\left(\mathcal{N}_{\left(g_{1}, g_{2}\right)}\right)\right] \wedge \mathfrak{T}\left(\mathcal{N}_{\left(g_{1} g_{2}\right)}, \Phi\right) \\
& =\sum_{\left\{\left(g_{1}, g_{2}\right)\right\}}\left(e_{12}\right)_{*}\left[e_{1}^{*} c h_{\Phi}\left(e_{\left(g_{1}\right)}^{*} \alpha_{1}\right) \wedge e_{2}^{*} c h_{\Phi}\left(e_{\left(g_{2}\right)}^{*} \alpha_{2}\right) \wedge e\left(E_{\left(g_{1}, g_{2}\right)}^{[2]}\right)\right. \\
& \left.\wedge \mathfrak{T}\left(E_{\left(g_{1}, g_{2}\right)}^{[2]} \oplus \mathcal{N}_{\left(g_{1}, g_{2}\right)}\right) \wedge e_{12}^{*} \mathfrak{T}\left(\mathcal{N}_{\left(g_{1} g_{2}\right)}, \Phi\right)\right] \\
& =\sum_{\left\{\left(g_{1}, g_{2}\right)\right\}}\left(e_{12}\right)_{*}\left[e_{1}^{*} \operatorname{ch}_{\Phi}\left(e_{\left(g_{1}\right)}^{*} \alpha_{1}\right) \wedge e_{2}^{*} c h_{\Phi}\left(e_{\left(g_{2}\right)}^{*} \alpha_{2}\right) \wedge e\left(E_{\left(g_{1}, g_{2}\right)}^{[2]}\right)\right. \\
& \left.e_{1}^{*} \mathfrak{T}\left(\mathcal{N}_{\left(g_{1}\right)}, \Phi\right) \wedge e_{2}^{*} \mathfrak{T}\left(\mathcal{N}_{\left(g_{2}\right)}, \Phi\right)\right] \\
& =\left(e_{12}\right)_{*}\left[e_{1}^{*} \widetilde{c h}_{\text {deloc }}\left(\alpha_{1}\right) \wedge e_{2}^{*} \widetilde{c h}_{\text {deloc }}\left(\alpha_{2}\right) \wedge e\left(E_{\left(g_{1}, g_{2}\right)}^{[2]}\right)\right. \\
& =\widetilde{c h}_{\text {deloc }}\left(\alpha_{1}\right) *_{\text {CR }} \widetilde{c h}_{\text {deloc }}\left(\alpha_{2}\right) \text {. }
\end{aligned}
$$

Here we apply the identity (4.12). This implies that $\widetilde{c h}_{\text {deloc }}$ is a ring homomorphism.

From Proposition 2.7, we know that ch deloc $: K_{\text {orb }}^{*}(\mathfrak{X}) \otimes \mathbb{C} \rightarrow H_{C R}^{*}(\mathfrak{X}, \mathbb{C})$ is an isomorphism of complex vector spaces. As the degree zero component of $\mathfrak{T}\left(\mathcal{N}_{e}, \Phi\right)$ is $1, \mathfrak{T}\left(\mathcal{N}_{e}, \Phi\right)$ is an invertible element of the ring $H_{C R}^{*}(\mathfrak{X}, \mathbb{C})$. So $\widetilde{c h}_{\text {deloc }}$ is also an isomorphism of complex vector spaces. Hence $\widetilde{c h}$ deloc is a ring isomorphism.

Remark 4.6. Note that $K_{\text {orb }}^{*}(\tilde{\mathfrak{X}})=\bigoplus_{(g)} K_{\text {orb }}^{*}\left(\mathfrak{X}_{(g)}\right), H^{*}(\tilde{\mathfrak{X}}, \mathbb{C})=\bigoplus_{(g)} H^{*}\left(\mathfrak{X}_{(g)}, \mathbb{C}\right)$, and

$$
c h_{\Phi}: \bigoplus_{(g)} K_{\text {orb }}^{*}\left(\mathfrak{X}_{(g)}, \mathbb{C}\right) \longrightarrow \bigoplus_{(g)} H^{*}\left(\mathfrak{X}_{(g)}, \mathbb{C}\right)
$$

preserves the decompositions. This decomposition preserving homomorphism motivates the following alternative definition of a stringy product which might simplify computation when applying Theorem 4.5.

The isomorphism ch deloc $: K_{\text {orb }}^{*}(\mathfrak{X}, \mathbb{C}) \longrightarrow \bigoplus_{(g)} H_{\text {orb }}^{*}\left(\mathfrak{X}_{(g)}, \mathbb{C}\right)$ induces the decomposition

$$
K_{\text {orb }}^{*}(\mathfrak{X}, \mathbb{C})=\bigoplus_{(g)} K_{\text {orb }}^{*}(\mathfrak{X},(g))
$$


such that $c h_{\text {deloc }}$ preserves the decomposition. Then the commutative triangle (4.6) immediately implies the following identities:

$$
\operatorname{ch}_{\Phi}\left(e_{(h)}^{*} \omega_{(g)}\right)= \begin{cases}\operatorname{ch}_{\operatorname{deloc}}\left(\omega_{(g)}\right) & \text { if }(g)=(h), \\ 0 & \text { otherwise }\end{cases}
$$

for any $\omega_{(g)} \in K_{\text {orb }}^{*}(\mathfrak{X},(g))$, and where $e_{(h)}: \mathfrak{X}_{(h)} \rightarrow \mathfrak{X}$ denotes the orbifold embedding. Define

$$
\bar{e}^{*}=\bigoplus_{(g)} e_{(g)}^{*}: K_{\text {orb }}^{*}(\mathfrak{X}, \mathbb{C})=\bigoplus_{(g)} K_{\text {orb }}^{*}(\mathfrak{X},(g)) \longrightarrow K_{\text {orb }}^{*}(\tilde{\mathfrak{X}}, \mathbb{C})=\bigoplus_{(g)} K_{\text {orb }}^{*}\left(\mathfrak{X}_{(g)}, \mathbb{C}\right)
$$

by sending $\alpha_{(g)} \in K_{\text {orb }}^{*}(\mathfrak{X},(g))$ to $e_{(g)}^{*} \alpha_{(g)} \in K_{\text {orb }}^{*}\left(\mathfrak{X}_{(g)}, \mathbb{C}\right)$. It is straightforward to check that $\bar{e}^{*}$ is a ring homomorphism preserving the decomposition. Moreover, the homomorphism $\bar{e}^{*}$ satisfies

$$
c h_{\text {deloc }}=c h_{\Phi} \circ \bar{e}^{*}
$$

and has a canonical left inverse $\bar{e}_{\#}$ defined in a similar way as in (4.8) - (4.10). We can check that the stringy product in Definition 4.3 can be written as

$$
\alpha_{1} \circ \alpha_{2}=\bar{e}_{\#}\left(\bar{e}^{*} \alpha_{1} \bullet A R Z \bar{e}^{*} \alpha_{2}\right)
$$

for $\alpha_{1}, \alpha_{2} \in K_{\text {orb }}^{*}(\mathfrak{X}, \mathbb{C})$. In particular, if $\alpha_{1} \in K_{\text {orb }}^{*}\left(\mathfrak{X},\left(g_{1}\right)\right)$ and $\alpha_{2} \in K_{\text {orb }}^{*}\left(\mathfrak{X},\left(g_{2}\right)\right)$, then

$$
\alpha_{1} \circ \alpha_{2} \in K_{\text {orb }}^{*}\left(\mathfrak{X},\left(g_{1} g_{2}\right)\right) .
$$

Example 4.7. The first example is the orbifold $[\{p t\} / G]$ given by a point $\{p t\}$ with a trivial action of a finite group $G$. The groupoid $\mathcal{G}$ associated to $[\{p t\} / G]$ is the action groupoid $G \rightrightarrows\{p t\}$ with the conjugation action groupoid $G \times G \rightrightarrows G$ as its inertia groupoid $\tilde{\mathcal{G}}$.

The Chen-Ruan cohomology ring of $[\{p t\} / G]$ is isomorphic to the center $Z(\mathbb{C}[G])$ of the group algebra $\mathbb{C}[G]$; see [14] or [2]. Alternatively, $H^{*}(\tilde{\mathcal{G}}, \mathbb{C})$ can be identified with the vector space of $\mathbb{C}$-valued $G$-conjugation invariant functions (also called class functions) on $G$. Let $\mathcal{C}(G)$ be the vector space of $\mathbb{C}$-valued class functions on $G$. The ordinary cup product on $H^{*}(\tilde{\mathcal{G}}, \mathbb{C})$ is the point-wise multiplication on $\mathcal{C}(G)$. As complex vector spaces, $\mathcal{C}(G)$ is isomorphic to the center $Z(\mathbb{C}[G])$ of the group algebra $\mathbb{C}[G]$. The Chen-Ruan product on $Z(\mathbb{C}[G])$ induces the convolution product on $\mathcal{C}(G)$,

$$
\left(\chi_{1} * \chi_{2}\right)(g)=\sum_{\left\{g_{1}, g_{2} \in G: g_{1} g_{2}=g\right\}} \chi_{1}\left(g_{1}\right) \chi\left(g_{2}\right),
$$

for $\chi_{1}, \chi_{2} \in \mathcal{C}(G)$, and $g \in G$. This means

$$
\left(H_{C R}^{*}([\{p t\} / G], \mathbb{C}), *_{C R}\right) \cong(\mathcal{C}(G), *) .
$$

The stringy product on the orbifold K-theory of the inertia orbifold was described in 4. Here we consider the stringy product on $K_{G}(\{p t\})$, the orbifold K-theory of the orbifold $[\{p t\} / G]$ itself. Note that

$$
K_{G}(\{p t\}) \cong R(G),
$$

the representation ring of $G$. The odd equivariant K-theory of a point vanishes. Let $\rho_{V}: G \rightarrow G L(V)$ be a representation of $G$. Then from the definition, $c h_{d e l o c}\left(\left[V, \rho_{V}\right]\right)$ is a class function on $G$ given by the character of the representation $\rho_{V}$,

$$
\operatorname{ch}_{\text {deloc }}([V, \rho])(g)=\operatorname{Tr}\left(\rho_{V}(g)\right), \quad \text { for } \quad g \in G,
$$


that is, ch deloc $: R(G) \rightarrow \mathbb{C}$ is a $\mathbb{C}$-valued class function on $G$. Then

$$
\text { ch deloc }: R(G) \otimes \mathbb{C} \longrightarrow \mathcal{C}(G)
$$

is a ring isomorphism, where the ring structure on $R(G) \otimes \mathbb{C}$ is given by the tensor product of representations and the ring structure on $\mathcal{C}(G)$ is the standard point-wise multiplication.

Let $\rho_{V}: G \rightarrow G L(V)$ and $\rho_{W}: G \rightarrow G L(W)$ be two representations of $G$. We compute the Adem-Ruan-Zhang product

$$
e^{*}\left[V, \rho_{V}\right] \bullet A R Z e^{*}\left[W, \rho_{W}\right]=\left(e_{12}\right)_{*}\left(e_{1}^{*} e^{*}\left[V, \rho_{V}\right] \otimes e_{2}^{*} e^{*}\left[W, \rho_{W}\right]\right),
$$

represented by a $G$-equivariant vector bundle over $G$, since there are no normal bundles involved in this case. As a vector bundle over $\tilde{\mathcal{G}}$, its fiber over $g \in G$ is

$$
\bigoplus_{\left\{g_{1}, g_{2} \in G: g_{1} g_{2}=g\right\}} V_{g_{1}} \otimes W_{g_{2}},
$$

where $V_{g_{1}}=V$ and $W_{g_{2}}=W$. The automorphism $\Phi$ on the fiber over $g$ is given by

$$
\bigoplus_{\left\{g_{1}, g_{2} \in G: g_{1} g_{2}=g\right\}} \rho_{V}\left(g_{1}\right) \otimes \rho_{W}\left(g_{2}\right) .
$$

By a direct calculation, we get

$$
\operatorname{ch}_{\Phi}\left(e^{*}\left[V, \rho_{V}\right] \bullet A R Z e^{*}\left[W, \rho_{W}\right]\right)=\operatorname{ch}_{\text {deloc }}\left(\left[V, \rho_{V}\right]\right) * \operatorname{ch} h_{\text {deloc }}\left(\left[W, \rho_{W}\right]\right),
$$

where $*$ is the convolution product on $\mathcal{C}(G)$. Therefore, the stringy product on $K_{\text {orb }}([\{p t\} / G], \mathbb{C})$ agrees with the convolution product on $\mathcal{C}(G)$ under the delocalized Chern character. Therefore, we get the ring isomorphism

$$
\left(K_{\text {orb }}^{*}([\{p t\} / G]) \otimes \mathbb{C}, \circ\right) \cong\left(H_{C R}^{*}([\{p t\} / G], \mathbb{C}), *_{C R}\right) .
$$

In summary, for the orbifold $[\{p t\} / G]$, the orbifold K-theory $K_{\text {orb }}^{*}([\{p t\} / G]) \cong$ $R(G)$ is isomorphic to the singular cohomology of its inertia orbifold over the complex coefficients under the delocalized Chern character. There are two products on $K_{\text {orb }}^{*}([\{p t\} / G], \mathbb{C})$.

(1) One is given by the tensor product, under the delocalized Chern character, corresponding to the point-wise product on $\mathcal{C}(G)$.

(2) The other is the stringy product, under the delocalized Chern character, corresponding to the convolution product on $\mathcal{C}(G)$.

Example 4.8. The orbifold is $[G / G]$, obtained by the conjugation action of a finite group $G$ on itself. The groupoid $\mathcal{G}$ for $[G / G]$ is the action groupoid of the $G$-conjugation action on $G$. Its inertia groupoid $\tilde{\mathcal{G}}$ is the action groupoid of the $G$-conjugation action on

$$
\tilde{G}=\{(g, h) \in G \times G \mid g h=h g\},
$$

the set of pairs of commuting elements in $G$.

Additively, $K_{\text {orb }}^{*}([G / G])$, the orbifold K-theory of $[G / G]$, is isomorphic to

$$
K_{G}(G) \cong \underset{\{(g) \text { :conjugacy classes of } G\}}{ } R\left(Z_{G}(g)\right),
$$

where $Z_{G}(g)$ is the centralizer of $g$ in $G$; see [4. An element in $K_{G}(G)$ is represented by a collection of finite-dimensional complex vector spaces $\left\{\left(V_{g}, \rho_{g}\right)\right\}_{g \in G}$, where 
$\rho_{g}: Z_{G}(g) \rightarrow G L\left(V_{g}\right)$ is a group homomorphism, such that there is a linear $G$ action on $\oplus_{g} V_{g}$ intertwining with the $\oplus \rho_{g}$-representation in the following sense:

(1) for any $k \in G$, there is a linear isomorphism $\phi_{V}(k): V_{g} \rightarrow V_{k g k^{-1}}$ satisfying $\phi_{V}\left(k_{1} k_{2}\right)=\phi_{V}\left(k_{1}\right) \circ \phi_{V}\left(k_{2}\right)$ for $k_{1}, k_{2} \in G$,

(2) $\phi_{V}(h)=\rho_{g}(h)$ for $h \in Z_{G}(g)$,

(3) the following diagram commutes:

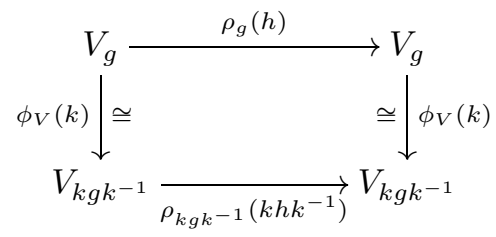

for any $h \in Z_{G}(g)$.

Diagram (4.19) implies that for $(g, h) \in \tilde{G}$,

$$
\operatorname{Tr}\left(\rho_{g}(h)\right)=\operatorname{Tr}\left(\rho_{k g k^{-1}}\left(k h k^{-1}\right) .\right.
$$

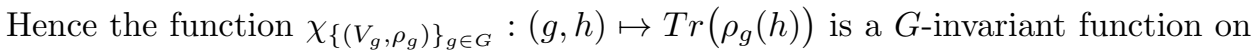
$\tilde{G}$. Let $\mathcal{C}_{G}(\tilde{G})$ be the space of $\mathbb{C}$-valued $G$-invariant functions on $\tilde{G}$. Then the delocalized Chern character

$$
c h_{\text {deloc }}: K_{G}(G) \longrightarrow \mathcal{C}_{G}(\tilde{G})
$$

is given by sending $\left\{\left(V_{g}, \rho_{g}\right)\right\}_{g \in G}$ to $\chi_{\left\{\left(V_{g}, \rho_{g}\right)\right\}_{g \in G}}$.

There are three different products on $K_{G}(G) \otimes_{\mathbb{Z}} \mathbb{C}$ described as follows:

(1) The first one is given by the usual tensor product $\otimes_{G}$ of $G$-equivariant vector bundles over $G$. The delocalized Chern character

$$
\text { ch deloc }:\left(K_{G}(G) \otimes \mathbb{C}, \otimes_{G}\right) \longrightarrow\left(\mathcal{C}_{G}(\tilde{G}), \cdot\right)
$$

is a ring isomorphism for the point-wise product $\cdot$ on $\mathcal{C}_{G}(\tilde{G})$.

(2) The second one is the Pontryagin product $\bullet_{G}$ given by

$$
\bullet_{G}: K_{G}(G) \times K_{G}(G) \stackrel{\pi_{1}^{*} \times \pi_{2}^{*}}{\longrightarrow} K_{G}(G \times G) \stackrel{m_{*}}{\longrightarrow} K_{G}(G),
$$

where $\pi_{1}, \pi_{2}: G \times G \rightarrow G$ are the obvious projections and $m: G \times G \rightarrow G$ is the group multiplication. This product $\bullet_{G}$ agrees with the Adem-RuanZhang product $\bullet A R Z$ on the orbifold K-theory of the inertia orbifold of $[\{p t\} / G]$. The ring $\left(K_{G}(G), \bullet_{G}\right)$, or its complexification, is the fusion ring for the three-dimensional topological quantum field theory associated to the finite gauge group $G$; see [18] and [19].

Explicitly, given two elements

$$
V=\bigoplus_{(g)}\left[V_{(g)}\right], \quad W=\bigoplus_{(g)}\left[W_{(g)}\right]
$$

in $K_{G}(G)=\bigoplus_{(g)} R\left(Z_{G}(g)\right)$, the Pontryagin product is given by

$$
\left\{(V \bullet G W)_{g}=\bigoplus_{g_{1}, g_{2} \in G: g_{1} g_{2}=g} V_{g_{1}} \otimes W_{g_{2}}\right\}_{g \in G}
$$

with the diagonal $G$-action

$$
\bigoplus \phi_{V}(k) \otimes \phi_{W}(k):\left(V \bullet_{G} W\right)_{g} \longrightarrow\left(V \bullet_{G} W\right)_{k g k^{-1}}
$$


for any $k \in G$. The delocalized Chern character

$$
\text { ch }_{\text {deloc }}: K_{G}(G) \otimes \mathbb{C} \longrightarrow \mathcal{C}_{G}(\tilde{G})
$$

is a linear isomorphism of vector spaces. For an abelian group $G, \tilde{G}=G \times G$. Then the Pontryagin product $\bullet_{G}$ induces the convolution product on $\mathcal{C}_{G}(\tilde{G})$ in the first variable:

$$
\left(f_{1} *_{1} f_{2}\right)(g, h)=\sum_{\left\{g_{1}, g_{2} \in G: g_{1} g_{2}=g\right\}} f_{1}\left(g_{1}, h\right) f_{2}\left(g_{2}, h\right) .
$$

(3) The third one is the stringy product $\circ$ on the orbifold K-theory of the orbifold $[G / G]$ itself defined in Definition 4.3. Given two elements

$$
V=\bigoplus_{(g)}\left[V_{(g)}\right], \quad W=\bigoplus_{(g)}\left[W_{(g)}\right]
$$

in $K_{\text {orb }}^{*}([G / G], \mathbb{C}) \cong \bigoplus_{(g)} R\left(Z_{G}(g)\right) \otimes \mathbb{C}$, the stringy product on $[G / G]$ is given by

$$
V \circ W=\bigoplus_{(g)}\left[V_{(g)} \circ Z_{G}(g) W_{(g)}\right]
$$

where $V_{(g)}{ }^{\circ} Z_{G}(g) W_{(g)}$ is the stringy product on $K_{\text {orb }}^{*}\left(\left[\{p t\} / Z_{G}(g)\right], \mathbb{C}\right)$ discussed in Example 4.7

The delocalized Chern character

$$
\text { ch deloc }:\left(K_{G}(G) \otimes \mathbb{C}, \circ\right) \longrightarrow\left(\mathcal{C}_{G}(\tilde{G}), *_{2}\right)
$$

is a ring isomorphism, where the product $*_{2}$ on $\mathcal{C}_{G}(\tilde{G})$ is given by

$$
\left(f_{1} *_{2} f_{2}\right)(g, h)=\sum_{\left\{h_{1}, h_{2} \in Z_{G}(g): h_{1} h_{2}=h\right\}} f_{1}\left(g, h_{1}\right) f_{2}\left(g, h_{2}\right) .
$$

For an abelian group $G, *_{2}$ is the convolution product on $\mathcal{C}_{G}(\tilde{G})$ in the second variable:

$$
\left(f_{1} *_{2} f_{2}\right)(g, h)=\sum_{\left\{h_{1}, h_{2} \in G: h_{1} h_{2}=h\right\}} f_{1}\left(g, h_{1}\right) f_{2}\left(g, h_{2}\right) .
$$

A simple example like $G=\mathbb{Z}_{2}$ shows that these three products are indeed different.

Corollary 4.9. For any finite group $G$, there is a ring isomorphism

$$
\left(H_{C R}([G / G], \mathbb{C}), *_{C R}\right) \cong\left(\mathcal{C}_{G}(\tilde{G}), *_{2}\right) .
$$

Example 4.10 (Weighted projective spaces). Consider the weighted projective space $W \mathbb{P}(p, q)$, where $p$ and $q$ are coprime integers, which can be presented as the quotient of the unit sphere $S^{3} \subset \mathbb{C}^{2}$ by the $S^{1}$-action

$$
e^{i \theta}\left(z_{1}, z_{2}\right)=\left(e^{i p \theta} z_{1}, e^{i q \theta} z_{2}\right) .
$$

As an orbisphere, $W \mathbb{P}(p, q)$ can be covered by two orbifold charts at singular points $x=[1,0]$ and $y=[0,1]$ with isotropy groups $\mathbb{Z}_{p}$ and $\mathbb{Z}_{q}$ respectively. Here $\mathbb{Z}_{w_{i}}$ is the cyclic subgroup of $S^{1}$ generated by the primitive $w_{i}$-th root of unity.

The orbifold K-theory of $W \mathbb{P}(p, q)$ over $\mathbb{C}$ was given in [3] as

$$
K_{\text {orb }}^{*}(W \mathbb{P}(p, q), \mathbb{C}) \cong \mathbb{C}[u] /\left\langle(1-u)^{2}\right\rangle \oplus \mathbb{C}\left(\zeta_{p}\right) \oplus \mathbb{C}\left(\zeta_{q}\right),
$$

where $\mathbb{C}\left(\zeta_{p}\right)$ and $\mathbb{C}\left(\zeta_{q}\right)$ are the $p$-th and $q$-th cyclotomic fields over $\mathbb{C}$. 
We can apply Theorem 4.5. Remark 4.6 and Example 4.28 in 2 to find the stringy product on $K_{\text {orb }}^{*}(W \mathbb{P}(p, q), \mathbb{C})$. Let

$$
\alpha_{p} \in \mathbb{C} \oplus \mathbb{C}\left(\zeta_{p}\right), \quad \beta_{q} \in \mathbb{C} \oplus \mathbb{C}\left(\zeta_{q}\right)
$$

be elements such that the delocalized Chern characters correspond to the constant function 1 on the twisted sectors corresponding to generators of $\mathbb{Z}_{p}$ and $\mathbb{Z}_{q}$ respectively. Then we have

$$
\alpha_{p}^{p}=\beta_{q}^{q}=1-u, \quad \alpha_{p}^{p+1}=\beta_{q}^{q+1}=0 .
$$

\section{TWisted CASES}

As explained in Section 4.1, the Adem-Ruan-Zhang stringy product is defined for twisted K-theory of the inertia orbifold of a compact, almost complex orbifold. In this section, we explain how the constructions in Section 4 can be carried over to torsion twisted cases.

A twisting $\sigma$ over an orbifold $\mathfrak{X}$ is a principal $P U(H)$-bundle over $\mathfrak{X}$. Then there exists an orbifold atlas such that $\sigma$ is represented by a $U(1)$-central extension of the canonically associated groupoid $\mathcal{G}[\mathcal{U}]=\left(\mathcal{G}_{1} \rightrightarrows \mathcal{G}_{0}\right)$. This central extension,

$$
U(1) \longrightarrow \mathcal{R} \longrightarrow \mathcal{G}_{1} \rightrightarrows \mathcal{G}_{0},
$$

called a $U(1)$-gerbe over $\left(\mathcal{G}_{1} \rightrightarrows \mathcal{G}_{0}\right)$, is obtained from the $P U(H)$-valued cocycle on $\mathcal{G}[\mathcal{U}]$ and the $U(1)$-central extension of $P U(H)$. Note that a gerbe connection is a connection $\theta$ on the $U(1)$-bundle $\mathcal{R}$ which is compatible with the groupoid multiplication on $\mathcal{R}$. A curving for the connection $\theta$ is a 2 -form $B$ on $\mathcal{G}_{0}$ such that the curvature of $\theta$ is given by

$$
F_{\theta}=s^{*} B-t^{*} B \in \Omega^{2}\left(\mathcal{G}_{1}\right) .
$$

Then $d B \in \Omega^{3}\left(\mathcal{G}_{0}\right)$ satisfies the property

$$
s^{*}(d B)-t^{*}(d B)=0 .
$$

Hence, the 3 -form $d B$ defines a closed 3 -form $\Omega$ on the orbifold $\mathfrak{X}$, called the gerbe curvature of the gerbe $\mathcal{R}$ with a gerbe connection $\theta$ and a curving $B$. A twisting $\sigma$ is called torsion if its associated gerbe is flat, that is, it has a gerbe connection and a curving whose gerbe curvature vanishes. By a standard construction (Proposition 3.6 in [43]), taking a refined orbifold atlas if necessary, the gerbe (5.1) always admits a gerbe connection and a curving.

The central extension (5.1) defines a complex line bundle over $\mathcal{G}_{1}$. Restricted to the inertia groupoid $\tilde{\mathcal{G}}$, we get a complex line bundle over the inertia groupoid, denoted by

$$
\mathcal{L}_{\sigma}=\bigsqcup_{(g) \in \mathcal{T}_{1}} \mathcal{L}_{(g)} \longrightarrow \bigsqcup_{(g) \in \mathcal{T}_{1}} \mathfrak{X}_{(g)} .
$$

When the gerbe is equipped with a gerbe connection and curving, property (5.2) implies that the induced connection on the complex line bundle $\mathcal{L}_{\sigma}$ is flat. Hence, $\mathcal{L}_{\sigma}=\left\{\mathcal{L}_{(g)}\right\}_{(g) \in \mathcal{T}_{1}}$ is an inner local system defined in [34] and 39]. Recall that an inner local system over an orbifold is a flat complex orbifold line bundle

$$
\mathcal{L}_{\sigma}=\bigsqcup_{(g) \in \mathcal{T}_{1}} \mathcal{L}_{(g)} \longrightarrow \bigsqcup_{(g) \in \mathcal{T}_{1}} \mathfrak{X}_{(g)}
$$


satisfying the following compatibility conditions:

(1) $\mathcal{L}_{(1)}$ is a trivial orbifold line bundle with a fixed trivialization.

(2) There is a non-degenerate pairing $\mathcal{L}_{(g)} \otimes I^{*} \mathcal{L}_{\left(g^{-1}\right)} \rightarrow \mathcal{L}_{(1)}$.

(3) There is an associative multiplication

$$
e_{\left(g_{1}\right)}^{*} \mathcal{L}_{\left(g_{1}\right)} \otimes e_{\left(g_{2}\right)}^{*} \mathcal{L}_{\left(g_{2}\right)} \longrightarrow e_{\left(g_{1} g_{2}\right)}^{*} \mathcal{L}_{\left(g_{1} g_{2}\right)}
$$

over $\mathfrak{X}_{\left(g_{1}, g_{2}\right)}$ for $\left(g_{1}, g_{2}\right) \in \mathcal{T}_{2}$. Here $e_{3}=I \circ e_{12}$.

Given an inner local system $\mathcal{L}_{\sigma}$ on an almost complex, compact orbifold $\mathfrak{X}$, the $\mathcal{L}_{\sigma}$-twisted Chen-Ruan cohomology is defined to be

$$
H_{C R}^{*}\left(\mathfrak{X}, \mathcal{L}_{\sigma}\right)=\bigoplus_{(g) \in \mathcal{T}_{1}} H^{d-2 \iota(g)}\left(\mathfrak{X}_{(g)}, \mathcal{L}_{(g)}\right)
$$

with the twisted Chen-Ruan product given by the same expression as in Definition 3.4. The properties of the inner local system ensure that the Poincaré pairing

$$
\left\langle\omega_{1}, \omega_{2}\right\rangle=\int_{\mathfrak{X}_{(g)}}^{o r b} \omega_{1} \wedge I^{*} \omega
$$

for $\omega_{1} \in H^{*}\left(\mathfrak{X}_{(g)}, \mathcal{L}_{(g)}\right)$ and $\omega_{2} \in H^{*}\left(\mathfrak{X}_{\left(g^{-1}\right)}, \mathcal{L}_{\left(g^{-1}\right)}\right)$ is non-degenerate, and the twisted Chen-Ruan product is determined by the the 3 -point function

$$
\left\langle\omega_{1} *_{C R} \omega_{2}, \omega_{3}\right\rangle=\int_{\mathfrak{X}_{\left(g_{1}, g_{2}\right)}}^{o r b} e_{1}^{*} \omega_{1} \wedge e_{2}^{*} \omega_{2} \wedge e_{3}^{*} \omega_{3} \wedge e\left(E_{\left(g_{1}, g_{1}\right)}^{[2]}\right),
$$

for $\omega_{1} \in H^{*}\left(\mathfrak{X}_{\left(g_{1}\right)}, \mathcal{L}_{\left(g_{1}\right)}\right), \omega_{2} \in H^{*}\left(\mathfrak{X}_{\left(g_{2}\right)}, \mathcal{L}_{\left(g_{2}\right)}\right)$ and $\omega_{3} \in H^{*}\left(\mathfrak{X}_{\left(g_{3}\right)}, \mathcal{L}_{\left(g_{3}\right)}\right)$ with $g_{3}=\left(g_{1} g_{2}\right)^{-1}$.

Let $\sigma$ be a torsion twisting on an almost complex compact orbifold $\mathfrak{X}$. We assume that the twisted orbifold K-theory defined by the Grothendieck group of $\mathcal{R}$-twisted vector bundles over $\mathfrak{X}$ agrees with the twisted K-theory defined in Section 4.1, Let $\check{\sigma}$ be the gerbe associated to $\sigma$ with a gerbe connection and curving. Note that if the gerbe curvature of $\check{\sigma}$ is zero, then the twisted Chern character on the twisted orbifold K-theory $K_{\text {orb }}^{*}(\mathfrak{X}, \sigma)$ constructed in [43],

$$
c h_{\check{\sigma}}: K_{\text {orb }}^{*}(\mathfrak{X}, \sigma) \otimes \mathbb{C} \longrightarrow H^{*}\left(\tilde{\mathfrak{X}}, \mathcal{L}_{\sigma}\right)=\bigoplus_{(g) \in \mathcal{T}_{1}} H^{*}\left(\mathfrak{X}_{(g)}, \mathcal{L}_{(g)}\right),
$$

is an isomorphism of vector spaces over the complex coefficients. Geometrically, this twisted Chern character is constructed as follows. Let $E$ be an $\mathcal{R}$-twisted vector bundle over $\mathcal{G}_{0}$. Then the pull-back vector bundle $e^{*} E$ over $\tilde{\mathcal{G}}_{0}$ admits a bundle isomorphism $\Phi$ defined by an element in $\operatorname{End}\left(e^{*} E\right) \otimes \mathcal{L}_{\sigma}$. With respect to a locally constant trivialization, the Chern character $c h_{\Phi}$ as in Section 4.2 can be extended to the twisted case to define a homomorphism

$$
\operatorname{ch}_{\Phi, \sigma}: K_{\text {orb }}^{*}\left(\tilde{\mathfrak{X}}, e^{*} \sigma\right) \longrightarrow H^{*}\left(\tilde{\mathfrak{X}}, \mathcal{L}_{\sigma}\right)
$$

such that the following diagram commutes:

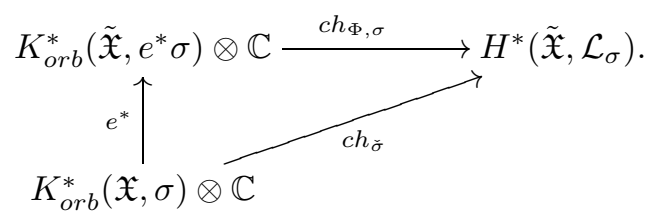


If $e^{*} \sigma$ is transgressive, then we can apply the Adem-Ruan-Zhang product on $K_{\text {orb }}^{*}\left(\tilde{\mathfrak{X}}, e^{*} \sigma\right)$ to define a stringy product on $K_{\text {orb }}^{*}(\mathfrak{X}, \sigma) \otimes \mathbb{C}$. Modifying the twisted Chern character in (5.5) as in Definition 4.4 and Theorem 4.5, we expect that $K_{\text {orb }}^{*}(\mathfrak{X}, \sigma) \otimes \mathbb{C}$ with this string product is isomorphic to the twisted Chen-Ruan cohomogy $H^{*}\left(\tilde{\mathfrak{X}}, \mathcal{L}_{\sigma}\right)$ using the Mayer-Vietoris exact sequence. We leave the details of the proof of this isomorphism to interested readers.

\section{ACKNOWLEDGMEnTs}

The authors would like to thank Yongbin Ruan for many valuable discussions during the course of this work. This work was partly supported by the NSFC Grant 10825105 (first author) and the ARC Discovery Grant (second author). The first author thanks MSI of the Australian National University and the second author thanks Sun Yat-Sen University for their hospitality during part of the writing of this paper. The authors would like to thank the referee for valuable suggestions that improved the expositions and Alex Amenta for his comments.

\section{REFERENCES}

[1] A. Adem, M. Kalus, Lectures on Orbifolds and Group Cohomology Advanced Lectures in Mathematics 16 (L.Ji and S-T Yau, editors), Transformation Groups and Moduli Spaces of Curves (2010), 1-17.

[2] A. Adem, J. Leida, Y. Ruan, Orbifolds and stringy topology, Cambridge Tracts in Mathematics 171, Cambridge University Press, 2007. MR2359514 (2009a:57044)

[3] A. Adem, Y. Ruan, Twisted orbifold K-theory, Comm. Math. Phys. 237 (2003), no. 3, 533-556. MR1993337 (2004e:19004)

[4] A. Adem, Y. Ruan, B. Zhang, A stringy product on twisted orbifold K-theory. Morfismos (10th Anniversary Issue), 11, no. 2 (2007), 33-64. ArXiv:0605534.

[5] M. F. Atiyah, R. Bott, A Lefschetz fixed point formula for elliptic complexes, I. Ann. of Math. (2), 86 (1968), 546-604. MR0212836 (35:3701)

[6] M. F. Atiyah, G. Segal, Twisted K-theory. Ukr. Math. Bull. 1 (2004), no. 3, 291-334. MR:2172633(2006m:55017)

[7] P. Baum, A. Connes, Geometric K-theory for Lie groups and foliations. Enseign. Math. (2), 46 (2000), no. 1-2, 3-42. MR.1769535 (2001i:19006)

[8] P. Baum, A. Connes, K-theory for discrete groups, in Operator Algebras and Applications, (D. Evans and M. Takesaki, editors), Cambridge University Press, 1989, 1- 20. MR.996437 (91i:46083)

[9] V. Batyrev, Birational Calabi-Yau n-folds have the equal betti numbers, New trends in algebraic geometry (Warwick, 1996), London Math. Soc. Lecture Note Ser., 264, Cambridge Univ. Press, Cambridge, 1999, pp. 1-11. math.AG/9710020. MR/1714818(2000i:14059)

[10] E. Becerra, B. Uribe, Stringy product on twisted orbifold K-theory for abelian quotients. Trans. Amer. Math. Soc. 361 (2009), no. 11, 5781-5803. MR2529914 (2011b:19008)

[11] J.-L. Brylinski, Loop Spaces, Characteristic Classes and Geometric Quantization. Progr. Math., vol. 107, Boston, MA, 1993. MR1197353 (94b:57030)

[12] J.-L. Brylinski, V. Nistor, Cyclic cohomology of étale groupoids, K-Theory 8 (1994), 341-365. MR 1300545 (96c:19001)

[13] B. Chen, S. Hu, A deRham model for Chen-Ruan cohomology ring of abelian orbifolds. Math. Ann., 336 (2006), no. 1, 51-71. Math.SG/0408265. MR2242619 (2007d:14044)

[14] W. Chen, Y. Ruan, A new cohomology theory of orbifold, Comm. Math. Phys. 248 (2004), no.1, 1-31. MR2104605 (2005j:57036)

[15] A. Connes, Noncommutative geometry. Academic Press Inc., San Diego, CA, 1994. MR.1303779 (95j:46063)

[16] D. Edidin, T. Jarvis, T. Kimura, Logarithmic trace and orbifold products. Duke Math. J. 153 (2010), no. 3, 427-473. MR2667422(2011f:14078) 
[17] B. Fantechi, L. Göttsche, Orbifold cohomology for global quotients. Duke Math. J. 117 (2003), no. 2197-227. MR1971293 (2004h:14062)

[18] D. S. Freed, Higher algebraic structures and quantization, Commun. Math. Phys. 159 (1994), 343-398. MR 1256993 (95c:58034)

[19] D. S. Freed, K-theory in quantum field theory, in Current developments in mathematics, Int. Press, Somerville, MA, 2001, pp. 41-87. MR1971377(2004b:58032)

[20] R. Goldin, M. Harada, T. S. Holm, T. Kimura, The full orbifold K-theory of abelian symplectic quotients, arXiv:0812.4964v2. To appear in Journal of K-theory. MR2842935

[21] A. Henriques, D. S. Metzler, Presentations of non effective orbifolds, Trans. Amer. Math. Soc., 356 (2004), no. 6, 2481-2499. MR2048526 (2005a:58027)

[22] R. Hepworth, The age grading and the Chen-Ruan cup product. Bull. Lond. Math. Soc. 42 (2010), no. 5, 868-878. MR2721746 (2012b:55008)

[23] M. Hilsum, G. Skandalis, Morphismes K-orientés d'espaces de feuilles et fonctorialité en théorie de Kasparov (d'après une conjecture d'A. Connes), Ann. Sci. École Norm. Sup. (4), 20 (1987), no. 3, 325-390. MR925720 (90a:58169)

[24] T. Jarvis, R. Kaufmann, T. Kimura, Stringy K-theory and the Chern character, Invent. Math. 168 (2007), no. 1, 23-81. ArXiv:math/0502280. MR2285746(2007i:14059)

[25] T. Kawasaki, The index of elliptic operators on V-manifolds. Nagoya Math. J., 9 (1981), 135-157. MR641150(83i:58095)

[26] B. Lawson, M-L, Michelsohn, Spin geometry. Princeton Mathematical Series, 38. Princeton University Press, Princeton, NJ, 1989. MR.1031992 (91g:53001)

[27] E. Lupercio, B. Uribe, Gerbes over orbifolds and twisted K-theory, Comm. Math. Phys. 245 (2004), no. 3, 449-489. MR2045679 (2005m:53035)

[28] I. Moerdijk, Orbifolds as groupoids: An introduction. In Orbifolds in mathematics and physics (Madison, WI, 2001), Contemp. Math., 301 (2002), 205-222. MR1950948 (2004c:22003)

[29] I. Moerdijk, J. Mrun, Introduction to foliations and Lie groupoids, Cambridge Studies in Advanced Mathematics. vol. 91, Cambridge University Press, Cambridge, 2003. MR2012261 (2005c:58039)

[30] I. Moerdijk, D. A. Pronk, Orbifolds, sheaves and groupoids, K-Theory 12 (1997), no. 1, 3-21. MR $1466622(98 \mathrm{i}: 22004)$

[31] I. Moerdijk, D. A. Pronk, Simplicial cohomology of orbifolds, Indag. Math. (N.S.) 10 (1999), no. 2, 269-293. MR1816220 (2002b:55012)

[32] M. K. Murray, Bundle Gerbes. J. Lond. Math. Soc. 54(1996), no. 2, 403-416. MR.1405064 (98a:55016)

[33] M. K. Murray, D. Stevenson, Bundle Gerbes. Stable isomorphism and local theory. J. Lond. Math. Soc. 62 (2007), no. 2, 925-937. MR1794295 (2001j:55019)

[34] J. Z. Pan, Y. B. Ruan, X. Q. Yin, Gerbes and twisted orbifold quantum cohomology. Sci. China Ser. A 51 (2008), no. 6, 995-1016. MR2410979 (2009d:53134)

[35] D. Quillen, Elementary proofs of some results of cobordism theory using Steenrod operations, Advances in Math. 7 (1971), 29-56. MR0290382 (44:7566)

[36] Y. Ruan, Stringy geometry and topology of orbifolds, in Contemp. Math., 312, Amer. Math. Soc., Providence, RI, 2002, pp. 187-233. MR.1941583 (2004b:32051)

[37] Y. Ruan, Stringy orbifolds. in Orbifolds in mathematics and physics (Madison, WI, 2001), Contemp. Math., 310, Amer. Math. Soc., Providence, RI, 2002, pp. 259-299. MR.1950951 (2004e:53136)

[38] Y. Ruan, The cohomology ring of crepant resolutions of orbifolds, in Gromov-Witten theory of spin curves and orbifolds, Contemp. Math., vol. 403, Amer. Math. Soc., Providence, RI, 2006, pp. 117-126. MR2234886 (2007e:14093)

[39] Y. Ruan, Discrete torsion and twisted orbifold cohomology. J. Symplectic Geom. 2 (2003), no. 1 1- 24. MR2128387(2006c:57027)

[40] Y. Ruan, Surgery, quantum cohomology and birational geometry, Northern California Symplectic Geometry Seminar, 183-198, Amer. Math. Soc. Transl. Ser. 2, 196, Amer. Math. Soc., Providence, RI, 1999. MR,1736218 (2001h:14074)

[41] I. Satake, On a generalization of the notion of manifold. Proc. Nat. Acad. Sci. U.S.A. 42 (1956), 359-363. MR0079769 (18:144a)

[42] W. Thurston, The geometry and topology of three-manifolds, Princeton Lecture Notes, 1980.

[43] J. Tu, P. Xu, Chern character for twisted K-theory of orbifolds. Advances in Mathematics, 207 (2006), 455-483. MR2271013 (2007h:58036) 
[44] J. Tu, P. Xu, C. Laurent-Gengoux, Twisted K-theory of differentiable stacks, Ann. Sci. École Norm. Sup. (4) 37 (2004), no. 6, 841-910. MR2119241(2005k:58037)

[45] C-L. Wang, On the topology of birational minimal models, J. Diff. Geom. 50 (1998), 129-146. MR:1678489(2000m:14012)

Department of Mathematics, Sun Yat-Sen University, Guangzhou, 510275, People's Republic of China

E-mail address: stsjxhu@mail.sysu.edu.cn

Department of Mathematics, Australian National University, Canberra aCt 0200, Australia

E-mail address: bai-ling.wang@anu.edu.au 